\title{
$\$$ Research Square

\section{A Pig White Matter Atlas and Common Connectivity Space Provide a Roadmap for the Introduction of a New Animal Model in Translational Neuroscience}

\section{R. Austin Benn}

Centro Nacional de Investigaciones Cardiovasculares (CNIC)

\section{Rogier Mars}

Wellcome Centre for Integrative Neuroimaging, Centre for Functional MRI of the Brain (FMRIB), Nuffield Department of Clinical Neurosciences, John Radcliffe Hospital, Oxford

\section{Ting Xu}

Child Mind Institute Inc

\section{Luis Rodríguez-Esparragoza}

Hospital Universitario Fundación Jimenez Diaz

\section{Paula Montesinos}

Philips Healthcare Iberia

\section{J.P Manzano-Patron}

University of Nottingham

\section{Gonzalo Lopez-Martin}

entro Nacional de Investigaciones Cardiovasculares (CNIC)

\section{Valentin Fuster}

Centro Nacional de Investigaciones Cardiovasculares (CNIC)

Javier Sánchez-González

Philips Healthcare Iberia

\section{Eugene Duff}

University of Oxford https://orcid.org/0000-0001-8795-5472

Borja Ibanez ( $\sim$ bibanez@cnic.es )

Spanish National Centre for Cardiovascular Research

\section{Article}

Keywords: Homology in the Cerebral Cortex, Translational Model, MRI, Tractography, Cortical Alignment

Posted Date: November 16th, 2020

DOI: https://doi.org/10.21203/rs.3.rs-105759/v1 
License: (c) (i) This work is licensed under a Creative Commons Attribution 4.0 International License. Read Full License 


\section{Abstract}

The characterization and definition of homology in the cerebral cortex needed for a species to be adopted as a translational model in neuroscience is a unique challenge given the diverse array of cortical morphology present in the mammalian lineage. Using the domestic pig as an example, we provide a roadmap of how leveraging Magnetic Resonance Imaging of the brain and data-driven tractography can overcome these obstacles and facilitate cortical alignment between distantly related species. In doing so, we created a full platform of neuroimaging tools to be used in the pig, including volumetric and surface templates, a structural white matter atlas, and the establishment of a common connectivity space to facilitate pig-human cortical alignment. Releasing our data and code and our pig-human cortical alignment, we permit researchers already working with the pig to accentuate the clinical relevance and translational capacity of their work. By sharing the intermediate outputs and scripts used to construct our pig-human cortical alignment, we also provide a roadmap to expand the current repertoire of animal models used in neuroscience.

\section{Introduction}

The introduction of a new animal model in neuroscience is challenging, given the vast body of work already performed in the well-established rodents and Non-Human primates (NHP). Mechanistic studies prefer rodents since they are easy to handle and relatively easy to modify genetically, but large animals' similar body size, organ shape, lifespan, and metabolism better model certain aspects of human disease. These advantages are not exclusive to NHPs, and large animal models, including pigs, dogs, and cats, all share a gyrencephalic brain, which can be studied using human imaging equipment. Years of tracer injections, imaging, and invasive recordings have provided a significant head start understanding the NHP cerebral cortex's organization. However, interest has recently grown in using the domestic pig (sus scrofa) as an alternative to NHP models ${ }^{1}$. We take advantage of this, and use the pig as an example of how the creation of volumetric and surface templates, a novel white matter atlas, and a first pass towards mapping the pig and human cortex via a common connectivity space enhance the translational capacity of underutilized animals in neuroscience.

The pig diverged from humans approximately 80 million years ago, but convergent evolution and an omnivore diet have led to a viscera strikingly similar to that of the human ${ }^{2,3}$. The pig can be genetically modified and is already used as a model for cardiac, renal, gastric, hepatic, dermal, vascular, craniofacial surgery, making it an ideal model to investigate the neural correlates of disease in the body ${ }^{4-10}$. Advances in high resolution non-invasive magnetic resonance imaging (MRI) and surgical methods have led to the development of stroke, deep brain stimulation, Parkinson's and Alzheimer's Disease, traumatic brain injury, and epilepsy models renewing interest in the pig as a neurological model of disease ${ }^{1,11-16}$. However, the translational value of these models is currently limited by a technical barrier, as no standardized resources or pipelines exist to process and contextualize pig imaging studies with the 
human brain. We overcome these limitations by creating a pig neuroimaging repository and developing a cross-species translational mapping between the pig and human.

The basis of our pig neuroimaging repository and the starting point for the translation of underutilized animals in neuroscience is creating a volumetric and surface standard template; In this case, the Porcine Neurological Imaging Space (PNI50) composed of 50 pigs. These templates are analogous to the commonly used Montreal Neurological Institute volumetric template (MNI152) and the Freesurfer average surface FSaverage ${ }^{17}$. Using anatomical and diffusion-weighted imaging (DWI), we then characterize the white matter structural organization in a subgroup of six pigs in an exploratory data-driven analysis (Figure $1 \mathrm{~A})^{18,19}$. Our exploratory analysis overcomes the scarcity of knowledge regarding the pig's white matter architecture and was used to guide the definition of hand-drawn tractography protocols in the PNI50 space for automated tractography in FSL autoPTX and Xtract ${ }^{20,21}$. We delineated 27 tracts to include in our WM atlas of the pig, including the projection, cross-hemispheric, association, and limbic tracts. With our tracts defined, we next built a connectivity blueprint ${ }^{22}$, extending a method previously used in the macaque to a non-primate. In doing so we exemplify the robustness of the connectivity blueprint method and its ability to use shared connectivity to cortically align distantly related species such as the pig and the human.

The connectivity blueprint is based on the simple idea that the shared connections to homologous structures can be used to characterize how similar an area is across species ${ }^{23,24}$. The individual unit of a connectivity blueprint is a diagnostic 'connectivity fingerprint' whereby the similarity of an area or region of interest's cortical connections to the underlying WM tracts is calculated to determine a connectivity profile which can be used to propose homology in distantly related species ${ }^{22-25}$. The connectivity blueprint is a collection of connectivity fingerprints for the whole cortex, whereby the WM connections of each vertex in the cortical surface are profiled to create a 'common connectivity space' describing the brains of both species (Figure 1B) ${ }^{22}$.

The shared connectivity space was built using the common tracts included in our pig WM atlas, and by calculating the Kullback-Liebler Divergence $(\mathrm{KL})$ distance metric between the pig and human connectivity blueprints. Using the KL similarity matrix to measure conserved cortical connectivity between species, we further show how it can be used to align the pig and human cortex to spatially predict of regions of interest across species. By releasing our connectivity blueprints, tract protocols, white matter atlas, and anatomical templates in the data and code release(https://github.com/neurabenn/pig_connectivity_bp_preprint), we provide examples of the resources needed to ease the adoption of a new animal model in translational neuroscience. We 
furthermore use the pig to provide insight into the organization of the mammalian cortex and enable experimental findings from pig disease models to be translated and used to form novel hypotheses of their associated neurological impact on the human brain.

\section{Results}

Template and Average Surface Construction: A standard reference space and template is necessary for the exchange, communication, and replicability of neuroimaging data and analysis. Using 50 male large white pigs weighing between $25-45 \mathrm{KG}$, we created the PNI50 template for spatial registration and normalization (Figure 2A). To allow for intuitive navigation of the pig brain, we registered the Saikali et al. cytoarchitectonic pig gray matter atlas into PNI50 space and converted it to an interactive XML atlas for use in FSLeyes so that users can interactively navigate and describe their results spatially in the pig. We also created the PNI50 average cortical surface, a surface analogous to Freesurfer's FsAverage for the pig (Figure $2 \mathrm{~B})^{17}$. These tools allow for mapping volumetric results and masks defined in the PNI50 volume to be projected onto the cortical surface. The PNI50, as a common brain space for the pig, permitted us to use data-driven methods to reconstruct tracts and further define standard masks for tractography protocols. All results described in the following sections are described in the PNI50 space.

\section{Exploratory Tractography used to Guide the Definition of Hand-drawn Tractography Protocols: A total of}

27 tracts were identified and are included in our WM atlas. Tractography was performed in probtrackx2 (using the option -omat2 ) ${ }^{26}$ with the PNI50 average mid-thickness surface used to seed from each vertex to a volumetric low-resolution whole-brain target mask (1.4mm isotropic). This resulted in a matrix of streamlines mapping each cortical vertex to every brain voxel ${ }^{18}$, which then underwent iterative Principal Components Analysis (iPCA), and Independent Components Analysis (ICA) (Figure 1A). We then linearly regressed the ICA spatial maps back into volumetric space, where they were visually assessed as plausible tracts for inclusion in the atlas ${ }^{18}$. Using the ICAs in volumetric space as a guide, we then drew seed and target masks by hand for tractography in the PNI50 standard space (Figure 3-6). In doing so, we created standard tractography protocols that recapitulate elements of the data-driven components for 27 tracts. The tractography protocols consist of a set of masks containing: the seed (tractography start point), target (waypoint, only streamlines that travel through here are retained), exclusion (areas prohibited to the streamline), and occasionally, stop (stops streamline propagation). All tractography protocols except for those of the cross-hemispheric structures use the sagittal midline as an exclusion mask to prevent streamline propagation into the contralateral hemisphere. These tract protocols allow for reproducible tractography of pigs registered to the common PNI50 space and are compatible with the recently released XTRACT package ${ }^{21}$. The repository we present here provides all 27 tractography protocols, the final tracts used to construct the connectivity blueprint, and the corresponding data-driven ICAs for each tract ${ }^{18,27}$. 
A White Matter Atlas of the Pig Brain: The 27 white matter tracts identified in the brains of 6 pigs were grouped by their respective systems: Projection, Cross-hemispheric, Associative, and Limbic. In the following section, we describe each tract group and the structures assigned to it, including each tract's course, the ICA used to guide protocol definition and the masks for each tractography protocol in the atlas. Tractography protocols were defined in PNI50 volume space and transformed into each pig's native DWI volume space where the tract was reconstructed. The resulting tracts were then transformed back to PNI50 space, where the group average normalized streamlines of 6 pigs were used to form the final tract in our probabilistic white matter atlas. In the absence of other gray matter atlases in the domestic pig, we used the Saikali et al. atlas for navigation and spatial characterization of our results ${ }^{27}$. We use their assignations as spatial guidelines for the reader but acknowledge that despite similar naming conventions, some labels may not indicate homology to structures in the human brain. However, conserved connectivity partially confirms the homology of some labels proposed in the Saikali et al. atlas (Figure 10,11).

Projection Fibers: Exploratory tractography found multiple components associated with the projection fibers and using them to guide the definition of hand-drawn protocols; the whole thalamus was used as the seed region for all four thalamic radiations presented here. Based on the principles of conserved cortical organization in the mammalian brain-plan ${ }^{28}$ we expected the thalamocortical projections and corticospinal tract to serve as a base for comparison of conserved connectivity across species.

Anterior Thalamic Radiation (ATR): Component 22 of the left hemisphere (L-22) and 45 in the right (R-45) contain a structure connecting the thalamus with the prefrontal cortex forming the ATR ${ }^{29}$ (Figure 3 ). Using these components to guide the definition of the tractography protocol for the ATR, the target mask was defined as the caudate nucleus, and a coronal stop mask was drawn below the thalamus at the level of the posterior commissure (Figure $3 \mathrm{~A}$ ).

Optic Radiation (OR): The optic radiation OR connects the inferior visual lobe with the thalamus and is present in components $\mathrm{L}-16 / 41$ and $\mathrm{R}-1 / 20$. The target mask is a coronal slice in the inferior junction of $\mathrm{V} 1$ and $\mathrm{V} 3$ at the occipitotemporal junction (Figure 3B).

Posterior Thalamic Radiation (PTR): The PTR runs superior to the OR and connects the thalamus to a V1 target mask. The PTR was present in components L-13/25/39 and R-6/15(Figure 3C). 
Superior Thalamic Radiation (STR): The Superior thalamic Radiation found in components L-17/36 and $\mathrm{R}-8 / 22 / 31$ radiates from the thalamus to the primary and somatosensory association cortex Figure $(7,8 \mathrm{D})$. The target is an axial section superior to the seed region, where the Primary/Associative Somatosensory Cortex meet. Coronal slices anterior the genu of the corpus callosum and at the posterior commissure are excluded (Figure 3D).

Corticospinal Tract (CST): The CST is partially found across components L-0/31/33/43 and R0/23/42/19 (Figure 3E). The CST connects the brainstem and sensorimotor cortex, replicating the structure of Bech et al. ${ }^{30}$. The CST seed is drawn inferior to the thalamus, and the target is an axial slice of the sensorimotor cortex. The exclusion mask has two coronal slices at the posterior commissure, the genu of the corpus callosum, and a third axial slice inferior to the thalamus that does not include the internal capsule (Figure 3E).

Commissural and cross-hemispheric Tracts: The commissural fibers and the cerebellar peduncle were found in components that span both hemispheres (Figure 4). All three tracts used a reverse seeding strategy whereby the target and seed were flipped in separate reconstructions, and the final tract is presented as their average. All three protocols largely replicate the tracts previously identified by Zhong et al. ${ }^{31}$.

Forceps Major (FMA): The FMA (components L-40 and R-20) connects the left and right visual cortices passing through the splenium of the corpus callosum ${ }^{31}$. The FMA was reverse seeded from V1 and V2 of each hemisphere, and a coronal section anterior the splenium of the corpus callosum was excluded (Figure 4A).

Forceps Minor (FMI): The FMI connects the left and right prefrontal cortex, as shown in components L$4 / 22$ and R-3/39 (Figure 4B). Coronal sections of the left and right dorsolateral prefrontal cortex are reverse-seed for the FMl, and there is no exclusion mask (Figure 4B).

Middle Cerebellar Peduncle (MCP): The MCP connects the left and right cerebellar peduncles and is found in components L-6/32 and R-42, which contain multiple tract structures ${ }^{31}$. The MCP used the left cerebellar peduncle as a seed and the right as the target (Figure 4C). 


\section{Association Fibers:}

Exploratory analysis identified three tracts with trajectories similar to the Inferior Fronto-Occipital Fasciculus, the Uncinate Fasciculus, and the Inferior Longitudinal Fasciculus of Pascalau et al.'s white matter dissection study (Figure 5) ${ }^{32}$. Components from data-driven tractography found structures reminiscent of the Superior Longitudinal Fasciculus (SLF) from Pascaleu et al.'s white matter dissection ${ }^{32}$. Our attempts to define a hand-drawn SLF tractography protocol were unsuccessful in replicating the SLF-like data-driven components, and we thus chose to leave it out of the pig white matter atlas for now.

Inferior Fronto-Occipital Fasciculus (IFOF): Exploratory tractography found multiple independent components containing IFOF-like structures connecting the visual and frontal cortex. In the left hemisphere, these structures were present in components 34 and 18 (L-34/18), and component 21 (R-21) in the right hemisphere (Figure 5A). These components guided the IFOF seed placement using a visual cortex composite mask of V1, V2, and V3. The target mask was a coronal slice of the anterior prefrontal cortex, and the external capsule and the ventricles were excluded in the axial plane (Figure 5A).

Inferior Longitudinal Fasciculus (ILF): A tract reminiscent of the primate ILF is present as a substructure in components L-7/9 and R-15/20 (Figure 5B). The ILF connects the inferior temporal gyrus to the inferior occipital lobe. The seed mask is placed at the middle/inferior temporal gyrus, and the target included the superior temporal gyrus in an axial section at the level of the zona incerta. Coronal slices anterior the seed and posterior to the target were excluded (Figure 5B).

Uncinate Fasciculus (UNC): The UNC connects the anterior region of the inferior temporal gyrus, arcs into the external capsule, and terminates in the Anterior Prefrontal Cortex. The UNC (L-48 and R-14/30) is seeded in the inferior temporal gyrus, where it targets the junction of the external capsule and putamen. The exclusion mask includes the visual cortex and the coronal plane around the target mask (Figure 5C).

Limbic Tracts: Data-driven tractography found components similar to fornix and cingulum, confirming the recent work of Bech et al. ${ }^{33}$. As in primates, the cingulum bundle tractography protocols were defined in three parts: the temporal, dorsal, and pregenual bundles. ICA components of the data-driven tractography found three distinct structures of similar course and length to the Cingulum proposed by Bech et al., suggesting their segmented reconstruction approach is necessary to reconstruct all three branches of the pig cingulum (Figure 6) ${ }^{33,34}$. We also note that our cingulum's cortical projections do not form a continuum but remain isolated within each segment. All protocols for the cingulum were reverse seeded. 
The fornix appears to be highly conserved across species as the primary hippocampal tract derived from data-driven components.

Cingulum Dorsal Bundle (CBD): The CBD can be found in components L-26 and R-28 coursing through the cingulate cortex superior to the corpus callosum as the central segment between the temporal and pregenual bundles (Figure $6 \mathrm{~A}$ ). The $\mathrm{CBD}$ is seeded in a coronal region of the dorsal posterior cingulate just above the splenium of the corpus callosum. The target mask lies at the border of the anterior and posterior cingulate cortex as defined in the Saikali et al. atlas, and the stop mask is placed just anterior to the target mask (Figure 6A). The tract passes cleanly through both seed and target mask, terminating at the border of the pregenual bundle.

Pregenual Cingulum (CBP): The CBP protocol is guided by components $\mathrm{L}-47$ and $\mathrm{R}-44$ and is seeded inferior to the genu of the corpus callosum with the CBD stop mask as its target (Figure 6B). The axial exclusion mask has a slice superior to the genu of the corpus callosum and a second axial slice at the level of the genu of the corpus callosum. A hole is left in the second slice of the exclusion mask, allowing streamlines to propagate throughout the Dorsal Anterior Cingulate. The tract enters the Dorsal Anterior Cingulate and passes the genu of the corpus callosum rostrally curving beneath it to terminate in the anterior prefrontal cortex.

Temporal Cingulum (CBT): The CBT is present in component R-11 running parallel to the fornix, and connects the dorsal posterior cingulate and parahippocampal cortex in concordance with the work of Bech et al. (Figure $6 \mathrm{C})^{33}$. The CBT was seeded in the parahippocampal cortex, with a target in the dorsal posterior cingulate, posterior the splenium of the corpus callosum ${ }^{33}$. The exclusion mask blocks the superior hippocampus, visual cortex, the genu, and the corpus callosum (Figure 6C). The stop mask is divided into two sections: one, anterior the target, and the second, posterior the fornix.

Fornix (Fx): The Fornix is included in the Saikali et al. atlas and is present in components L-01 and R$36 / 41$ (Figure 6D) $27,31,33$. The fornix is seeded at its apex as defined in the Saikali et al. atlas and runs inferior to the corpus callosum through the hippocampus and amygdala terminating in the parahippocampal area anterior to the CBT. The coronal target mask is in the inferior hippocampus, and the exclusion mask has two coronal slices: one posterior the caudate and the second posterior the genu of the corpus callosum (Figure 6D). 


\section{Connectivity Blueprints:}

The Connectivity Blueprint approach depends on the proposal of homologous white matter tracts in a 'common space' to compare the brain's structural organization across species (Figure 1B). The connections of a tract to the cortex form a cortical tract projection. When the cortical projections of multiple tracts are stored and normalized, a probabilistic distribution of the tracts with connections to a given point on the cortical surface forms a connectivity fingerprint (Figure 1B). When we calculate the connectivity fingerprint for each vertex of the cortical surface to its underlying WM tracts, we create a connectivity blueprint, which can be used to compare structural connectivity across the whole cortex for two brains (Figure 1B). Calculating the Kullback-Liebler divergence (KL) of the connectivity blueprint, we can measure the similarity of the connectivity fingerprint for each vertex in the brain. In doing so, we can identify regions with conserved structural connectivity (low KL) and those where structural organization has diverged between species (high KL).

Prior to the calculation of $\mathrm{KL}$ divergence between the pig and human, we first took the mean of the connectivity blueprint to create a whole cortex connectivity fingerprint in order to assess the symmetry of each species connectivity. We found the pig had greater connectivity in the left hemisphere to the FMI and CBP, whereas the STR and PTR displayed a more robust connectivity profile with the right hemisphere (Figure 7A). Furthermore, the overall connectivity profile as compared with the human brain found greater connectivity to the IFOF, ILF, and CBD in the human, as opposed to increased connectivity of the UNC, STR, and PTR in the pig (Figure 7B).

Between the pig and human, we calculated four cross-species blueprints whereby tract groups were added sequentially to the blueprint to understand each tract group's role in the formation of unique connectivity fingerprints for each species. The tract groups were added to the connectivity blueprint in the following order: projection fibers, commissural fibers, associative fibers, and limbic fibers. The order tract groups were added into the connectivity blueprint started with the assumption that the diverse trajectories of the projection tracts would provide an initial starting point to compare across the whole cortex. The commissural, associative, and limbic fibers were then added based on a separate leave one out analysis which determined which tract group most contributed to the $\mathrm{KL}$ divergence of the full connectivity blueprint (Supplementary Figure1). By plotting the tracts sequentially, we visualize the evolution of divergence between tract structures and their overall impact in forming distinct connectivity profiles between the pig and human (Figure 8). We further visualize the impact each tract group plays in the $\mathrm{KL}$ distribution through a leave-one-out analysis and the calculation of the difference in $\mathrm{KL}$ divergence between the full $\mathrm{KL}$ divergence, and the connectivity blueprints where a tract group has been left out (Figure S1,2). Both our sequential tract addition, and the leave-one-out analysis converge in identifying the regions most impacted by each tract group(Figure $8, \mathrm{~S} 1$ ). 
In the following section, we discuss the effect of the addition of each tract group had on the distribution of the minimum KL divergence between the pig and human, as explained by each tract's cortical projections. We also map each blueprint's entropy to determine the diversity of tracts connecting to any given vertex. We see that the sequential $K L$ divergence evolves not due to the number of tracts in each blueprint, but rather through the addition of specific WM structures.

Blueprint 1, The Projection Tracts: The lowest similarity between species, as shown by the highest KL divergence, corresponds with the tract projections of the ATR and CST (Figure 8A). The lateral division of the prefrontal and somatosensory cortices in the pig leads to partial innervation of the pig's anterior somatosensory cortex by the ATR. The cortical projections of the pig CST extend into the anterior frontal lobe overlapping with the projections of the ATR, whereas in the human, they remain within the pre and post-central gyrus (Figure 8A). The overlap between the CST and ATR of the pig causes an increase of unique connectivity fingerprints in the pig's frontal lobe where the local minimum of KL-divergence peaks(Figure 8A, S1A.2). Increased KL-divergence is further observed in regions associated with the PTR and OR given the increased presence of the human cortical projections of the temporal lobe (Figure 8A, S1A.1). Key to interpreting the local-minimum of $\mathrm{KL}$ divergence is the non-convergence with the entropy maps, representing the diversity of tracts connecting reaching a point on the cortex. This implies that the minimum $\mathrm{KL}$ divergence is dependent on the presence of distinct connectivity fingerprints as opposed to the number of tracts connecting to each point. Of all the tracts in blueprint one, the cortical projections of the STR appear to be the most conserved. The pig's projection fibers connect to a higher proportion of the cortex, but their relative organization appears to be conserved (Figure 7B,8). We do note that the overall role of the projection fibers in driving $\mathrm{KL}$ divergence is significant (Figure S2.A,E), however we start our sequential addition with them as their diverse trajectories provide a basis for comparing connectivity across the whole cortex of both species.

Blueprint 2, BP1 + The Commissural Tracts: $K L$ divergence increases minimally, suggesting that the similarity between species of the commissural tracts is minimal. However, where it does increase is in regions innervated by the FMA and FMI. The cortical projections of the FMA are conserved as they enter the medial occipital lobe in both species, and similarly, the MCP is conserved through a lack of projections to the cortex in both species (Figure 8B). The FMI does increase KL divergence in the lateral prefrontal cortex of the human and the medial-inferior frontal lobe of the pig (Figure 8B, S1A) . However, the FMI's cortical projection does not cross the lateral barrier into the somatosensory cortex, making it spatially conserved within the prefrontal cortex (Figure 8B). Contributing to the frontal lobe's increased KL divergence observed in both species is likely the higher connectivity to the whole cortex of the FMI in the human as compared to the pig (Figure 7B, ). Notably, where the medial FMI and FMA tract projections terminate, entropy decreases in both species. This appears to impact the KL divergence in these regions 
minimally (Figure S2, B,F)but accentuates the $\mathrm{KL}$ divergence observed in the territory of the projection fibers.

Blueprint 3, BP2 + Association Tracts: Adding the association fibers into the blueprint, the maximum of the min-KL divergence of the pig brain shifts to the prefrontal cortex and anterolateral somatosensory cortex (Figure 8C, S1C.2). Conversely, the highest KL divergence in the human cortical surface shifted to the Angular/Supramarginal Gyri, and lateral prefrontal cortex (Figure 8C, S1C.1). If we assume the pig's tract coursing from the occipital to the frontal cortex to be the IFOF, we still observe that changes in the $\mathrm{KL}$ divergence are associated with each species' distinct IFOF projection patterns. The pig's IFOF projects to the cortical surface in the anterior prefrontal cortex and occipital lobe, but lacks the temporoparietal projections found in the human IFOF (Figure 8 C). Furthermore, the human IFOF innervates a far greater portion of the cortex, increasing the probability that any given point of the human cortex connects to the IFOF compared to the pig, as shown through both the whole cortex connectivity fingerprint (Figure 7), and increased entropy in the frontal and parietal lobes of the human brain (Figure 8C). The UNC charts a course similar to that of the human but shows increased $\mathrm{KL}$ divergence in the temporal pole and inferior frontal cortex. The tract's cortical projections remain close to the tract starting in the inferior temporal gyrus and end in the inferior frontal lobe. The pig's UNC appears elongated as compared to that of the human (Figure 8C). The pig's lack of anterolateral expansion in the temporal lobe causes the pig ILF projection to run horizontally from the inferior to superior temporal gyrus, likely contributing to the increased $\mathrm{KL}$ divergence in the inferior temporal lobe of the pig (Figure S1C). In the human brain, where significant anterolateral expansion of the temporal lobe has taken place compared to the pig, the ILF's cortical projection runs diagonally in order to connect similar regions (Figure 8C).

The IFOF, ILF, and UNC share structural characteristics across both species. However, the increased KL divergence over the whole cortex signifies that these tracts' addition introduced a greater proportion of non-mutual connectivity fingerprints between the pig and human as compared to blueprints 1 and 2 . The association tracts contribute to the $\mathrm{KL}$ divergence in the human brain more so than in the pig (Figure $S 2 C, G)$. This can likely be attributed to the association tracts extensive cortical projections in the human brain as compared to those of the pig (Figure 7, 8C).

Blueprint 4, BP 3 + Limbic Tracts: Blueprint 4 contains all 27 tracts having added the Cingulum fibers and fornix (Figure 8D). Among the limbic tracts, the fornix has the most similar connectivity and cortical projection pattern, suggesting a highly conserved structure along the mammalian lineage (Figure 8D). However, the $\mathrm{KL}$ divergence is high within the medial frontal lobe and cingulum; the territory of the CBD, 
CBP, and CBT(Figure 8D, S1D). The pig lacks the characteristic continuity typically associated with the cingulum. This is shown by the cortical projection of the pig CBD arcing upwards connecting the precuneus and somatosensory/premotor area complex instead of extending anteriorly into the territory of the CBP as in the human (Figure 8D). These changes in cortical projections are reflected in the KL divergence of the leave one out groups, as well as the entropy maps of the sequential blueprints (Figure $8 \mathrm{D}, \mathrm{S} 2 \mathrm{D}, \mathrm{H})$. The addition of the limbic tracts causes the entropy in the human cortex to drop along the cingulum bundle but not in the pig. As the continuum of the cingulum consists of the tract fibers projecting to one another, it is logical that the diversity of tracts in this region decreases when added into the human blueprint, but without the continuums presence in the pig the proportion of shared connectivity fingerprints decreases causing the KL divergence to rise (Figure 8D, S1D).

Connectivity blueprints can predict neural correlates between the pig and human. Using the full $\mathrm{KL}$ Divergence matrix from blueprint 4, we predicted known regions of interest defined in the Harvard/Oxford MNI152 atlas ${ }^{35}$ onto the pig cortex. Using masks of the frontal pole, occipital pole, and precentral gyrus, we predicted matches to the Saikali et al. atlas of the dorsolateral prefrontal cortex, primary somatosensory cortex, and V1 of the visual cortex (Figure 9). We then predicted the Saiakli et al. labels implicated in the first prediction and found reasonable alignment to the previously used ROIs from the Harvard/Oxford atlas (Figure 10). Two-way predictions across species demonstrate the connectivity blueprint as a translational framework capable of interpreting neuroimaging derived results from the pig to the human. Of course, we do not claim that pig and human frontal pole are homologous, as it is known that parts of this part of the human brain are a unique expansion in that lineage ${ }^{36}$, but rather that results can be translated to areas that have similar connectivity fingerprints.

\section{Discussion}

The pig is widely used in biomedical research but lacks the established study of cortical organization and fundamental tools that have made non-human primates the premier large animal model in neuroscience today ${ }^{37}$. Bridging this gap, we show that characterizing the pig brain's structural organization and sharing the tools we have created can habilitate the use of an underutilized animal model by the neuroimaging community. Leveraging comparative MRI, we created a pig-human cortical alignment based on conserved structural organization of the pig and human neocortex. We enable the prediction of neural correlates across species with 80 million years of evolutionary divergence ${ }^{3}$. Given the recent development of deep brain stimulation, Parkinson's, Alzheimer's, and stroke models in the pig ${ }^{13-15}$, the tools presented here have the potential to permit findings from these models to inform the design of novel therapeutic strategies in the treatment of neurological disease. Furthermore the open distribution of our translational imaging and analysis platform will further allow for the neurological phenotyping of disease progression in the cardiac, renal, gastric, hepatic, and vascular disease models already available in the pig ${ }^{4-10}$. Doing so may provide insight into the adaptive responses and structural reorganization of the cortex initiated by 
disease in the body and could facilitate the combination of longitudinal studies in pigs and crosssectional studies in clinical subjects. This would permit us to study disease through the lens of cortical reorganization in a translational testing environment of new clinical hypotheses.

The PNI50 MRI T1 weighted anatomical template serves is as a common space for pigs and is the building block by which the application of conventional techniques commonly used in human neuroimaging can be applied to the pig. The corresponding average surface acts as the seed used in the data-driven tractography and allows for the projection of volumetric data and results onto the cortical surface. The volumetric and surface template files are included in the data and code release (https://github.com/neurabenn/pig_connectivity_bp_preprint), opening our data-driven and protocolbased tractography methods to be used by other researchers. All protocols were defined in the PNI50 and form part of an open resource for pig researchers compatible with FSL's Xtract and autoPtx ${ }^{20,21}$. Researchers working with pigs can register their data to the PNI50 and apply cross-species prediction, as demonstrated in figures 9 and 10. We note that as with the use of new methods in human neuroimaging, the open release of data and code for new animal models can significantly accelerate their adoption by the neuroimaging community ${ }^{38}$.

Using the PNI50 volumetric and surface templates, we next aimed to create standard definitions of the pig's white matter tracts. We identified a total of 27 tracts using a combination of data-driven and hypothesis based tractography. Combining both approaches to tractography overcomes the scarcity of knowledge regarding the WM organization common in underutilized models like the pig. Of the 27 tracts, 3 cross the medial wall, and 12 show relative symmetry in each hemisphere (Figure 7A). We extended the connectivity blueprint approach to a non-primate species in the pig. We used the blueprint to assess the impact the projection, commissural, association, and limbic tract groups had on conserved connectivity between the pig and human (Figure 8, S1,S2). We found the association and limbic tracts increased the overall $\mathrm{KL}$ divergence between both brains, suggesting that the areas innervated by these tracts are the least conserved (Figure 8C,D, S2).

The full connectivity blueprint containing 27 tracts was used to predict atlas regions of the precentral gyrus, frontal and occipital poles from the human to pig and vice versa. In the following section, we discuss similarities and differences found in the visual, motor, limbic, frontal, and temporal regions of the pig brain and how they define its experimental niche in translational models relevant to human health in neuroscience. 
Tracts of the Visual Cortex: The pig's lateral eye placement has led to the development of a panoramic field of vision that extends $310^{\circ}$ degrees on each side of the pig, and differs from the primate's forwardlooking visual system, which prioritizes binocular vision and depth perception ${ }^{39,40}$. Given these stark differences, we were surprised by the low $\mathrm{KL}$ divergence in the visual cortex and conserved connectivity. Tracts innervating this region included the IFOF, ILF, PTR, OR, and FMA. We did not find a tract similar to the Ventral Occipital Fasciculus in the pig, which likely impacted our mapping of similarity in the occipital lobe as connectivity blueprints can only account for tracts present in both species. The high degree to which structural connectivity was conserved in distinct visual systems was a surprise suggesting further functional characterization of the pig brain may be needed to highlight the organizational differences between the occipital lobe of the pig and human (Figure 8).

The tracts of the Somatosensory and Motor Cortex: The human division between the somatosensory and frontal lobes is in the anterior-posterior axis along the pre and postcentral gyri. The pig prefrontal and somatosensory cortex division is lateral across the coronal sulcus in the frontal lobe ${ }^{41}$. The somatosensory and motor cortex are innervated by the STR and CST in both species. However, the lateral division of the prefrontal and somatosensory cortex is noted as the CST and STR extend anteriorly into the pig's frontal lobe, and the ATR enters not only the lateral prefrontal cortex but also the anterior somatosensory cortex (Figure 7). The Gottingen minipig's prefrontal cortex was previously defined by the presence of the ATR with an injected tracer ${ }^{42}$. Our ATR structure replicates the tracer derived structure, but the ATR cortical projection's entrance into the anterior somatosensory cortex surprised us as it is typically considered a defining feature of the prefrontal division (Figure 8A). The proximity of the ATR to the CST and STR cortical projections suggests that studying the functional organization of the pig may provide novel insight into potentially unique communication pathways between a laterally separated prefrontal and somatosensory cortex not present in the primate lineage. Despite these differences, our cross-species alignment accurately mapped the pig somatosensory cortex, and human pre-central gyrus to one another (Figure 9C,10C).

The tracts of the Limbic System: The limbic system tracts were reconstructed with data-driven methods, and our mask protocols produced similar structures to the recent work of Bech et al. ${ }^{33}$ From our observations, the fornix appears to be the best conserved tract in the limbic system as the primary tract of the hippocampus. Unlike in the human, the cingulum tracts of the pig do not form a continuum as evidenced through the discontinuous tract projections of the CBD, CBP, and CBT. The pig's CBT extends outwards from the dorsal posterior cingulate toward the parahippocampal area (Figure 6, 8D) in concordance with the study of Bech et al., but we would expect a homologous CBT to terminate closer to the fornix in the amygdala ${ }^{43}$. We found further divergence in the CBP's cortical projection, which overlapped with the projection of the forceps minor contrasting the human CBP, which projects backward into the dorsal cingulum bundle. The CBD of the pig fails to project into either the pregenual or temporal 
bundles preventing the continuum of structural connectivity that unites the CBD, CBP, and CBT in the human; this is reflected in a clear pattern of high $\mathrm{KL}$ divergence and distinct entropy patterns along the cingulum (Figure $8 \mathrm{D}$ ). The CBD, CBP, and CPT's partial derivation by data-driven methods and their correspondence to the work of Bech et al. suggest their necessity in the connectivity blueprint to map divergence between the pig and human brain, especially as it appears the limbic tracts most drive the KL divergence between both species (Figure S2D,H).

The tracts of the Temporal Lobe: The pig's temporal lacks the anterolateral expansion characteristic of the temporal lobe in primates. A clear difference is marked as the rhinal fissure divides the temporal lobe in the pig from the main corpus of the cerebrum as opposed to the Sylvian fissure, a structure proposed to be unique to the primate lineage ${ }^{41,44}$. Despite a different size and structure of the temporal lobe, both species share the presence of ILF, and UNC-like structures, but unlike the human, the projections of the visual tracts (OR and PTR) do not extend into the temporal lobe (Figure 8A, C). The pig's IFOF enters the superior temporal gyrus, but its projections remain contained within the external capsule contrasting the IFOF's widespread temporal and parietal projections observed in the human (Figure 3,8A). Given the lack of homology between the pig and human temporal lobe, it would be premature to claim the pig's IFOF as a homologous structure in both brains. However, it is notable that a recent study on 130 mammalian species demonstrated a ventral longitudinal tract is present in most species, suggesting this is a common feature in the mammalian brain ${ }^{45}$. We did not attempt a mapping in the temporal lobe between the pig and human, as given the limited number of shared tracts in this area, it is unlikely sufficient landmarks exist to map it accurately.

The tracts of the Prefrontal Cortex: The pig's prefrontal cortex lacks a granular layer IV, but has tract projections to the medial dorsal thalamus as shown via tracer injections and our reconstruction of the Anterior Thalamic Radiation (Figure $8 \mathrm{~A})^{42}$. In addition to the ATR, the IFOF, FMI, and UNC show relatively conserved trajectories in both species. Unique connectivity fingerprints emerge in the lateral prefrontal cortex with the addition of the association tracts, likely due to the presence of the non-conserved connections of the human IFOF to the parietal and temporal lobe (Figure 8D, S1C). Notably, the morphology of the Uncinate Fasciculus of the pig is flatter and possesses a distinct angular orientation as compared to the human. This is likely due to the minimal anterolateral expansion present in the pig's temporal lobe (Figure 5). Our sequential blueprint, and leave one out analysis, further show that the greatest differences found in the frontal lobe follow the addition of the limbic tracts, and in particular, the tracts of the cingulum (Figure 8D, S1D, S2D,H). The CBP's extension into the territory innervated by the FMA causes the highest proportion of unique connectivity fingerprints in the medial prefrontal cortex, driving a marked increase in $\mathrm{KL}$ divergence (Figure 8D, S2D). 
The success of Cross-Species prediction: The pig and human brains share similar tracts, but that are tailored to the specific needs of each species. Despite profound changes in tract structure and cortical projections of the cingulum, and association tracts (especially the IFOF), we validate the connectivity blueprint approach by predicting the spatial coordinates of human regions of interest onto the pig cortex. This reverse-translation from human-to-pig overcame the differences in connectivity described above, and we successfully predicted the human frontal pole, visual cortex, and somatosensory cortex to their spatial coordinates within the pig brain (Figure 9,10). These predictions lined up with the work of Saikali et al. validating their cytoarchitecture atlas via conserved connectivity ${ }^{27}$. The success this cross-species cortical alignment provides a powerful tool for researchers working with the pig to contextualize their findings with the human brain, and further shows that connectivity blueprints can be used to characterize the cortex of lesser-studied species. The blueprints used to map across species included 27 tracts, including those least similar to that of the human, suggesting that identifying conserved regions between distantly related species requires the mapping of both difference and similarity between brains.

\section{Limitations:}

The tracts presented here are generally considered symmetrical in the human brain, and without the Superior Longitudinal (SLF), and Arcuate Fasciculus in both blueprints, cortical symmetry of connectivity may be overestimated in both species (Figure 7). While we did find data-driven components suggesting SLF-like structures and Pascalau et al. identify an SLF in a pig blunt dissection study, we were unable to reconstruct the SLF with a hand defined tractography protocol, leading us to leave it out of the final connectivity blueprint ${ }^{32}$. Post mortem study at high resolution as opposed to the in-vivo $1.4 \mathrm{~mm}$ scans we performed might also provide a more precise delineation of the pig's cingulum, and detangle the overlapping cortical projections of the IFOF and UNC in the external capsule. Finally, we rely on the Saikali et al. cytoarchitectural atlas for the initial characterization of each tract's start and endpoint. In the future, data-driven or metric registrations may provide a more accurate starting point for identifying potential homologous structures across a wide array of species $^{27,46,47}$.

Although we have sought to match tracts based on their similarity in their course and termination points, the labels we use here are merely suggestive of homology. Our approach uses suggested homology to investigate similarity and difference across brains, but it can also be used to test explicit hypotheses of homology. For instance, Roumazeilles et al. showed that when defining one branch of the human ILF as homologous to macaque ILF led to a greater overall divergence score than when definition another branch as homologous ${ }^{47}$. This suggests that only one of the human ILF branches is similar to that of the macaque. A similar approach can be employed to formally test homology of all proposed tracts here. However, it should be noted that the labeling of tracts with similar names is only the start of the investigation in the present approach and indeed we can show that some tracts whose homology is more 
certain, such as the corticospinal tract and the optic radiation lead to blueprints with relatively low divergence scores.

\section{Conclusions}

Using the pig as an example we exemplify how neuroimaging and a digital platform of open resources can habilitate underutilized animals as translational models in neuroscience. The large degree to which the pig is already used in biomedical research will advance our understanding of the neural correlates of well-established translational disease models in the pig. The digital and open nature of the tools and resources presented here demonstrates the importance of data-sharing to accelerate the acceptance of a new animal model as researchers currently working with pigs can now use the connectivity blueprints and resources provided here to contextualize their findings with the human brain. Finally, despite large scale morphological changes in the spatial organization of the pig and human brain, connectivity is relatively conserved, suggesting these methods can provide inference into conserved patterns of cortical organization in distantly related species.

\section{Methods}

Animal Preparation and MRI Acquisition: The institutional animal review board approved all experiments, and all imaging was performed on a Philips 3T Achieva scanner (The Best, Netherlands). Pigs were anesthetized with a cocktail of Ketamine $(20 \mathrm{mg} / \mathrm{kg})$, Midazolam $(0.5 \mathrm{mg} / \mathrm{kg})$, and Xylazine $(0.2 \mathrm{mg} / \mathrm{kg})$, and scanned lying in the prone position with a 32 channel cardiac coil.

Structural T1 Image Acquisition: A T1 weighted 3D Flash image was acquired (TR 10 ms, TE 4.8 ms, Flip Angle(FA) $10^{\circ}$, FOV $210 \mathrm{~mm}$, Matrix $264 \times 238,150$ slices at $0.8 \mathrm{~mm}$ isotropic resolution) was acquired for each pig.

DWI Image Acquisition: Diffusion-weighted data was acquired with 64 encoding directions and a single B0 image (TR 13,500 ms, TE 100.5 ms, Flip Angle (FA) 10', FOV 210 mm, Matrix 148 x, 150 slices, Slice Thickness 70, Slice Gap 0, Resolution $1.4 \mathrm{~mm}$ isotropic). Field map correction was done with a blip phase-encoded image of the same geometry and acquisition parameters as the Diffusion-weighted image.

Volumetric Construction of the PNI50 template: A total of 50 adolescent male pigs weighing between 25$50 \mathrm{Kg}$ were used to construct a T1 anatomical template containing the full field of view. Pigs were initially registered linearly using FSL's FLIRT to a single subject using 12 degrees of freedom(dof) ${ }^{48,49}$. Following 
initial alignment, the antsMultivariateTemplate.sh script was run to create the "full-body" anatomical template ${ }^{50}$. Following the creation of the full-body $\mathrm{T} 1$ template, a brain mask was defined in the template space. This mask was warped to all 50 pigs, where the brain was masked for extraction. The resultant images were then used to create a brain specific template using the same process as in the "full-body" template.

Average Surface Construction: Individual surfaces were first reconstructed using the precon_all pipeline (https://github.com/neurabenn/precon_all). Precon_all is a fully automated surface reconstruction pipeline for non-standard animal models in neuroimaging. Full-body images were input, and brain extraction was performed via registration to the PNI50 volumetric template space. Images were bias field corrected with Ants N4 and segmented using FSL's FAST, before running a modified version of Freesurfer's surface generation pipeline $17,48,51$. Individual surfaces were then used to create the group average surfaces within the precon_all library using a modified version of the Freesurfer make_average_surface script. The average surface, originally reconstructed with 10,242 vertices was then downsampled to 10,001 vertices to match the dimensions of the Mars et al. blueprint. ${ }^{22}$

Preprocessing of DWI data: DWI images were first corrected for susceptibility induced distortions with FSL topup, and then corrected for movement and eddy current off-resonance effects in FSL eddy 52,53. Following these initial steps, DWI images were rigidly registered to their anatomical images in FSL FLIRT with 6 dof ${ }^{49}$. The linear registration was concatenated to the nonlinear warp FSL FNIRT generated in the brain extraction process of precon_all ${ }^{54}$. The concatenated warp was then inverted, and the standard brain mask resampled into the native DWI space of each pig to allow for brain extraction. Following brain extraction, we prepared the data for probabilistic tractography by running a modified version of the preprocessing steps included in the autoPtx automated tractography pipeline ${ }^{20}$. This included the generation of deterministic tensors and Fractional Anisotropy images using DTIfit, and was followed by a probabilistic two-fiber tensor model estimation in Bedpost $X^{20,26,55}$.

\section{Tractography}

Data-Driven tractography: Using the average mid-thickness cortical surface in the left and right hemispheres as a seed, and a low resolution $(1.4 \times 1.4 \mathrm{~mm})$ whole-brain mask, we generated a matrix of streamlines passing from each vertex to every voxel in the brain. This was done through the probtrackx2, -omatrix 2 option, and a step length of 0.35 . After doing this for all 6 pigs, we performed iterative principal components analysis (iPCA) on the group average cortex $x$ brain matrix generated in the previous step. The results of iPCa were then fed into an Independent Components Analysis with a dimensionality set to 50 to identify exploratory tracts in the surface space. Linear regression then mapped the components on 
the surface space back into volumetric space, whereby they were saved and used to guide the tractography protocol definition. This was done with the Moriarty and lookatmoriarty MATLAB scripts from the Mr Cat toolbox (www.neuroecologylab.org) ${ }^{18,19}$ (Figure 1A). We do note that we only identified 27 tracts in our pig WM atlas, despite setting the dimensionality of the ICA to 50 components. However, given the nature of ICA to split components, it was common to find ICAs which contained only part of what was likely the full tract structure. On the other hand, it was also common for ICAs derived in this manner to contain multiple tracts such as in the case of the ILF, and FMA. All 50 ICAs of the left and right hemisphere are available as part of data and code release(https://github.com/neurabenn/pig_connectivity_bp_preprint) for visual inspection and for guidance in defining future tractography protocols.

Tractography using mask protocols: Using both the Saikali et al. Atlas and the data-driven components, we defined tract protocols in the PNI50 space, which could be used with either the autoPtx and Xtract packages ${ }^{20,21}$. These packages allow for tractography masks defined in a standard space to be warped to a subject's individual space for tract reconstruction using probtrackx, significantly streamlining the process of identifying tracts across multiple subjects. Using the default options of probtrackx in autoPtx, we successfully reconstructed 27 tracts in 6 pigs. We then took each individual's tract and transformed it back into the PNI50 space, where the average of all normalized streamlines was taken as the final group tract for the WM atlas.

Connectivity Blueprint Generation: The connectivity blueprint is a cortical surface' tract matrix describing the connectivity fingerprint between each vertex of the grey/white matter surface, and the tracts it connects to (figure 1B). The connectivity blueprint is created by the multiplication of a cortex $x$ brain matrix, and a tracts by brain matrix ${ }^{22}$. The group cortex $x$ brain matrix was generated in step one of the data-driven tractography and is the matrix that iPCA is applied to. The tract $x$ brain matrix is formed by a second tractography where each tract is used as a seed, and the same low-resolution mask from datadriven tractography is the target once again with the -omat2 option specified in probtrackx. The mean of this output is taken and added to a matrix containing the mean tract to brain connections of all tracts included in the blueprint to form the final tracts $x$ brain matrix. The connectivity blueprint for each hemisphere was then generated by multiplying the tract' brain and cortex ' brain matrices producing a matrix where each column contains the cortical projections of each tract (figure 1B, 8) ${ }^{22}$.

\section{Human Connectivity Blueprints:}

Human connectivity blueprints and surfaces were obtained from Mars et al. 's original connectivity blueprint paper (https://git.fmrib.ox.ac.uk/rmars/comparing-connectivity-blueprints) ${ }^{22}$. 
Identifying the tract Groups differentiating the pig and human: The porcine connectivity blueprints contain 27 tracts, and the human blueprint had 39 tracts. All normalization and calculations of KL divergence were performed as in Mars et. $\mathrm{al}^{22}$. Using a script in python (code release), we removed all tracts in the human blueprint that were not present in the pig. Having equally sized blueprints, we were then able to calculate $\mathrm{KL}$ divergence between the pig and human cortex. $\mathrm{KL}$ divergence functions as a measure of relative entropy between two given probability distributions and informs us about the amount of information lost between the two distributions as measured in units of bits. Lower KL divergence means less information is lost, and thus greater similarity between both distributions. In this case, mapping the minimum KL divergence over the human and pig surface provides a visual representation of regions where the connectivity fingerprints between the pig and human are the most, and least similar, where high $\mathrm{KL}$ signifies significant organizational changes between species.

Connectivity Blueprints consist of a collection of connectivity fingerprint of the whole cortex where the probability of each vertex connecting to each white matter tract is recorded. To better understand the contribution of each WM structure to the overall $\mathrm{KL}$ divergence, we plotted the mean probabilistic distribution of each blueprint, which quickly showed the relative proportion each tract connects to the cortex ( Figure 7). This allowed us to quickly infer which WM tracts were likely to be less conserved between species, and led us to add tract groups consecutively to the connectivity blueprints so we could individually assess the effect a tract group had on driving changes in $\mathrm{KL}$ divergence calculated between the pig and human cortex. All calculations for the consecutive KL divergence of tract groups are available data and code release(https://github.com/neurabenn/pig_connectivity_bp_preprint) as part of an interactive Jupyter notebook (Figure 8).

Cross-Species Prediction of Regions of Human Interest: Using the connectivity blueprint containing all 27, we calculated the $\mathrm{KL}$ similarity matrix for every cortical vertex between species. Regions of interest were then projected to their respective surface, and inverse weighted distance interpolation was applied using code modified from the original Mars et al. connectivity blueprint paper (Figure 9,10) ${ }^{22}$.

\section{Declarations}

Acknowledgements: We would like to thank the CNIC veterinary and animal facility staff, as well as Carlos Galán for their help in animal procurement and preparation. We would also like to thanks Fidel Alfaro, Steve Smith, and Michael P. Milham for their introduction to our collaborators at FMRIB, and the Child Mind Institute. RAB would like to thank Angel Macías and Braulio Perez for their technical support. 


\section{Funding:}

Ministry of Science and Innovation (MCN; "RETOS 2019" grant No PID2019-107332RB-I00), Instituto de Salud Carlos III (ISCIII; PI16/02110), and Comunidad de Madrid (S2017/BMD-3867 RENIM-CM). B.I is recipient of a European Research Council grant MATRIX (ERC-COG-2018-ID: 819775). RAB was supported by a fellowship from the FP7-PEOPLE-2013-ITN. "Cardionext". ED received funding from SSNAP "Support for Sick and Newborn Infants and their Parents" Medical Research Fund (University of Oxford Excellence Fellowship). RBM is supported by the Biotechnology and Biological Sciences Research Council (BBSRC) UK [BB/N019814/1]. The Wellcome Centre for Integrative Neuroimaging is supported by core funding from the Wellcome Trust [203139/Z/16/Z].

The CNIC is supported by the ISCIII, the MCN and the Pro CNIC Foundation and is a Severo Ochoa Center of Excellence (SEV-2015-0505).

\section{Author contributions:}

RAB: contributed to Conceptualization, Methodology, Data Acquisition, Formal analysis, Visualization, Writing - original draft, Writing - review \& editing; RBM: contributed to Conceptualization, Methodology, Formal analysis, Writing - review \& editing; TX: contributed to Methodology, Analysis, Visualization, Writing - review \& editing,; LRE: contributed to Methodology, Data Acquisition, Formal analysis; PM: contributed to Methodology, Data Acquisition; JPM: contributed to Data Acquisition, Formal analysis, Writing - review \& editing; GLM: contributed to Data Acquisition; VF: contributed to Writing -review \& editing, JGS: contributed to Conceptualization, Data Acquisition, Visualization, Writing - review \& editing; ED: contributed to Conceptualization, Methodology, and Writing - review \& editing; BI: contributed to Conceptualization, Methodology, Data Acquisition, Writing - review \& editing;

\section{Competing Interests:}

JSG and PM are employees of Philips Healthcare. All other authors have reported that they have no conflicts of interest related to the contents of this paper.

\section{Materials \& Correspondence:}


Materials and correspondence can be directed to either Borja Ibañez bibanez@cnic.es or R. Austin Benn r.austinbenn@gmail.com.

\section{References}

1. Lind, N. M. et al. The use of pigs in neuroscience: Modeling brain disorders. Neurosci. Biobehav. Rev. 31, 728-751 (2007).

2. Meurens, F., Summerfield, A., Nauwynck, H., Saif, L. \& Gerdts, V. The pig: A model for human infectious diseases. Trends Microbiol. 20, 50-57 (2012).

3. Kumar, S., Stecher, G., Suleski, M. \& Hedges, S. B. TimeTree: A Resource for Timelines, Timetrees, and Divergence Times. Mol. Biol. Evol. (2017). doi:10.1093/molbev/msx116

4. Chade, A. R., Williams, M. L., Engel, J., Guise, E. \& Harvey, T. W. A translational model of chronic kidney disease in swine. Am. J. Physiol. - Ren. Physiol. (2018). doi:10.1152/ajprenal.00063.2018

5. Gonzalez, L. M., Moeser, A. J. \& Blikslager, A. T. Porcine models of digestive disease: The future of large animal translational research. Translational Research (2015). doi:10.1016/j.trsl.2015.01.004

6. Kobayashi, E., Hishikawa, S., Teratani, T. \& Lefor, A. T. The pig as a model for translational research: Overview of porcine animal models at Jichi Medical University. Transplant. Res. 1, 1 (2012).

7. Dorado, B. et al. Generation and characterization of a novel knockin minipig model of HutchinsonGilford progeria syndrome. Cell Discov. (2019). doi:10.1038/s41421-019-0084-z

8. Martínez-Milla, J. et al. Translational large animal model of hibernating myocardium: characterization by serial multimodal imaging. Basic Res. Cardiol. (2020). doi:10.1007/s00395-0200788-0

9. Galán-Arriola, C. et al. Serial Magnetic Resonance Imaging to Identify Early Stages of AnthracyclineInduced Cardiotoxicity. J. Am. Coll. Cardiol. (2019). doi:10.1016/j.jacc.2018.11.046

10. Al-Mashhadi, R. H. et al. 18Fluorodeoxyglucose Accumulation in Arterial Tissues Determined by PET Signal Analysis. J. Am. Coll. Cardiol. (2019). doi:10.1016/j.jacc.2019.06.057

11. Sauleau, P., Lapouble, E., Val-Laillet, D. \& Malbert, C.-H. The pig model in brain imaging and neurosurgery. Anima/ 3, 1138 (2009).

12. Mäkiranta, M. et al. BOLD signal increase preceeds EEG spike activity - A dynamic penicillin induced focal epilepsy in deep anesthesia. Neuroimage 27, 715-724 (2005).

13. Zhang, R., Bertelsen, L. B., Flø, C., Wang, Y. \& Stødkilde-Jørgensen, H. Establishment and characterization of porcine focal cerebral ischemic model induced by endothelin-1. Neurosci. Lett. 635, 1-7 (2016).

14. Min, H. K. et al. Deep brain stimulation induces BOLD activation in motor and non-motor networks: An fMRI comparison study of STN and EN/GPi DBS in large animals. Neuroimage 63, 1408-1420 (2012).

15. Holm, I. E., Alstrup, A. K. O. \& Luo, Y. Genetically modified pig models for neurodegenerative disorders. Journal of Pathology (2016). doi:10.1002/path.4654 
16. Clouard, C., Jouhanneau, M., Meunier-Salaün, M. C., Malbert, C. H. \& Val-Laillet, D. Exposures to conditioned flavours with different hedonic values induce contrasted behavioural and brain responses in pigs. PLoS One 7, (2012).

17. Fischl, B. FreeSurfer. Neurolmage (2012). doi:10.1016/j.neuroimage.2012.01.021

18. O'Muircheartaigh, J. \& Jbabdi, S. Concurrent white matter bundles and grey matter networks using independent component analysis. Neuroimage 170, 296-306 (2018).

19. Mars, R. B. et al. Concurrent analysis of white matter bundles and grey matter networks in the chimpanzee. Brain Struct. Funct. 224, 1021-1033 (2019).

20. De Groot, M. et al. Improving alignment in Tract-based spatial statistics: Evaluation and optimization of image registration. Neuroimage (2013). doi:10.1016/j.neuroimage.2013.03.015

21. Warrington, S. et al. XTRACT - Standardised protocols for automated tractography and connectivity blueprints in the human and macaque brain. bioRxiv 804641 (2019). doi:10.1101/804641

22. Mars, R. B., Sotiropoulos, S. N. \& Passingham, R. E. Whole brain comparative anatomy using connectivity blueprints. Elife 245209 (2018). doi:10.1101/245209

23. Passingham, R. E., Stephan, K. E. \& Kötter, R. The anatomical basis of functional localization in the cortex. Nat. Rev. Neurosci. 3, 606-616 (2002).

24. Mars, R. B., Passingham, R. E. \& Jbabdi, S. Connectivity Fingerprints: From Areal Descriptions to Abstract Spaces. Trends in Cognitive Sciences 22, 1026-1037 (2018).

25. Mars, R. B. et al. Comparing brains by matching connectivity profiles. Neurosci. Biobehav. Rev. 60, 90-97 (2016).

26. Behrens, T. E. J., Berg, H. J., Jbabdi, S., Rushworth, M. F. S. \& Woolrich, M. W. Probabilistic diffusion tractography with multiple fibre orientations: What can we gain? Neuroimage (2007). doi:10.1016/j.neuroimage.2006.09.018

27. Saikali, S. et al. A three-dimensional digital segmented and deformable brain atlas of the domestic pig. 192, 102-109 (2010).

28. Krubitzer, L. The magnificent compromise: cortical field evolution in mammals. Neuron 56, 201-208 (2007).

29. Dyrby, T. B. et al. Validation of in vitro probabilistic tractography. Neuroimage 37, 1267-1277 (2007).

30. Bech, J. et al. The porcine corticospinal decussation: A combined neuronal tracing and tractography study. Brain Res. Bull. 142, 253-262 (2018).

31. Zhong, J. et al. An in vivo multi-modal structural template for neonatal piglets using high angular resolution and population-based whole-brain tractography. Front. Neuroanat. 10, 1-10 (2016).

32. Pascalau, R. \& Szabo, B. Fibre Dissection and Sectional Study of the Major Porcine Cerebral White Matter Tracts. 46, 378-390 (2017).

33. Bech, J. et al. Ex vivo diffusion-weighted MRI tractography of the Göttingen minipig limbic system. Brain Struct. Funct. (2020). doi:10.1007/s00429-020-02058-x 
34. Heilbronner, S. R. \& Haber, S. N. Frontal cortical and subcortical projections provide a basis for segmenting the cingulum bundle: Implications for neuroimaging and psychiatric disorders. $\mathrm{J}$. Neurosci. 34, 10041-10054 (2014).

35. Desikan, R. S. et al. An automated labeling system for subdividing the human cerebral cortex on MRI scans into gyral based regions of interest. Neuroimage (2006). doi:10.1016/j.neuroimage.2006.01.021

36. Neubert, F. X., Mars, R. B., Thomas, A. G., Sallet, J. \& Rushworth, M. F. S. Comparison of Human Ventral Frontal Cortex Areas for Cognitive Control and Language with Areas in Monkey Frontal Cortex. Neuron 81, 700-713 (2014).

37. Bolker, J. A. Selection of Models: Evolution and the Choice of Species for Translational Research. Brain. Behav. Evol. 93, 82-91 (2019).

38. Matthew F. Glassera, Stamatios N Sotiropoulosb, J Anthony Wilsonc, Timothy S Coalsona, Bruce Fischld, e, Jesper L Anderssonb, Junqian Xuf, g, Saad Jbabdib, Matthew Websterb, Jonathan R Polimenid, David C Van Essena, Mark Jenkinsonb, and for the W.-M. H. C. The Minimal Preprocessing Pipelines for the Human Connectome Project. (2017). doi:10.1016/j.neuroimage.2013.04.127.The

39. Grandin, T. Pig behavior studies applied to slaughter-plant design. Appl. Anim. Ethol. (1982). doi:10.1016/0304-3762(82)90190-0

40. Roelfsema, P. R. \& Treue, S. Basic neuroscience research with nonhuman primates: A small but indispensable component of biomedical research. Neuron 82, 1200-1204 (2014).

41. Bjarkam, C. R., Glud, A. N., Orlowski, D., Sørensen, J. C. H. \& Palomero-Gallagher, N. The telencephalon of the Göttingen minipig, cytoarchitecture and cortical surface anatomy. Brain Struct. Funct. 222, 2093-2114 (2017).

42. Jelsing, J. et al. The prefrontal cortex in the Göttingen minipig brain defined by neural projection criteria and cytoarchitecture. Brain Res. Bull. 70, 322-336 (2006).

43. Thiebaut, M., Schotten, D., Dell, F., Valabregue, R. \& Catani, M. Monkey to human comparative anatomy of the frontal lobe association tracts. CORTEX 48, 82-96 (2012).

44. Bryant, K. L. \& Preuss, T. M. A Comparative Perspective on the Human Temporal Lobe. (2018). doi:10.1007/978-4-431-56582-6

45. Assaf, Y., Bouznach, A., Zomet, O., Marom, A. \& Yovel, Y. Conservation of brain connectivity and wiring across the mammalian class. Nat. Neurosci. doi:10.1038/s41593-020-0641-7

46. Eichert, N. et al. What is special about the human arcuate fasciculus? Lateralization, projections, and expansion. Cortex 118, 107-115 (2019).

47. Roumazeilles, L. et al. Longitudinal connections and the organization of the temporal cortex in macaques, great apes, and humans. PLoS Biol. (2020). doi:10.1371/journal.pbio.3000810

48. Jenkinson, M., Beckmann, C. F., Behrens, T. E. J., Woolrich, M. W. \& Smith, S. M. FSL. Neurolmage 62, 782-790 (2012). 
49. Jenkinson, M., Bannister, P., Brady, M. \& Smith, S. Improved optimization for the robust and accurate linear registration and motion correction of brain images. Neuroimage (2002). doi:10.1016/S10538119(02)91132-8

50. Avants, B. B., Tustison, N. \& Song, G. Advanced Normalization Tools (ANTS). Insight J. 1-35 (2009).

51. Tustison, N. J. et al. N4ITK: Improved N3 bias correction. IEEE Trans. Med. Imaging (2010). doi:10.1109/TMI.2010.2046908

52. Andersson, J. L. R., Skare, S. \& Ashburner, J. How to correct susceptibility distortions in spin-echo echo-planar images: Application to diffusion tensor imaging. Neuroimage (2003). doi:10.1016/S1053-8119(03)00336-7

53. Andersson, J. L. R. \& Sotiropoulos, S. N. An integrated approach to correction for off-resonance effects and subject movement in diffusion MR imaging. Neuroimage(2016). doi:10.1016/j.neuroimage.2015.10.019

54. Andersson, J. L. R., Jenkinson, M. \& Smith, S. Non-linear registration aka spatial normalisation. FMRIB Technical Report TRO7JA2 (2007).

55. Behrens, T. E. J. et al. Characterization and Propagation of Uncertainty in Diffusion-Weighted MR Imaging. Magn. Reson. Med. (2003). doi:10.1002/mrm.10609

\section{Figures}
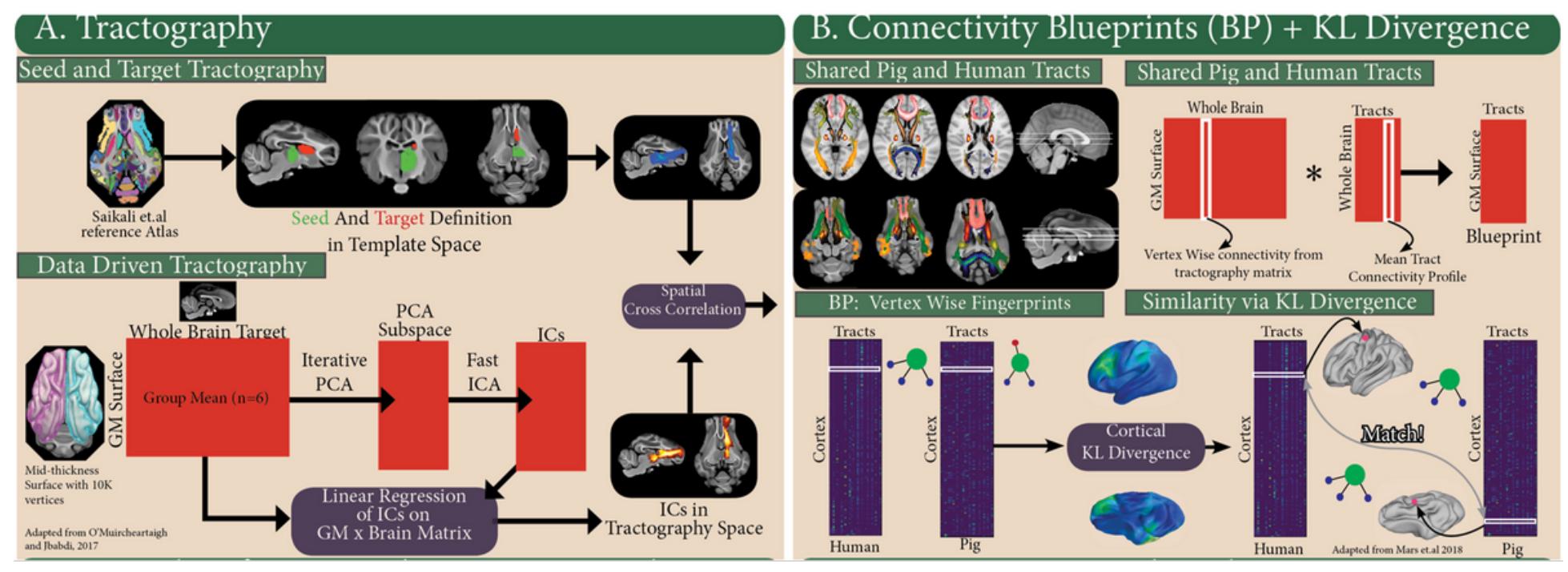

\section{Figure 1}

An overview of the methods used to produce the White Matter Tracts and subsequent Connectivity Blueprints. (A) Data-driven tractography uses a gray matter surface seeding to a low-resolution target. PCA is then run on the resulting tractography matrix, followed by ICA in the PC subspace, and linear regression returns the tracts to their volumetric space. Tractography protocols were then defined using information gained from the resulting ICAs, and masks were drawn in the PNI50 space using the Saikali et al. atlas. (B) Identifying common tracts between the pig and human, we calculated how each tract 
connected to each vertex in the mid-thickness cortical surface. We then created a connectivity blueprint whereby the connectivity profile of each vertex to all the common tracts was stored in each row, and the tract cortical projection each column. The pig blueprint was used with a human blueprint with proposed common tracts, and a KL divergence similarity matrix was calculated to identify regions with the highest similarity across species.
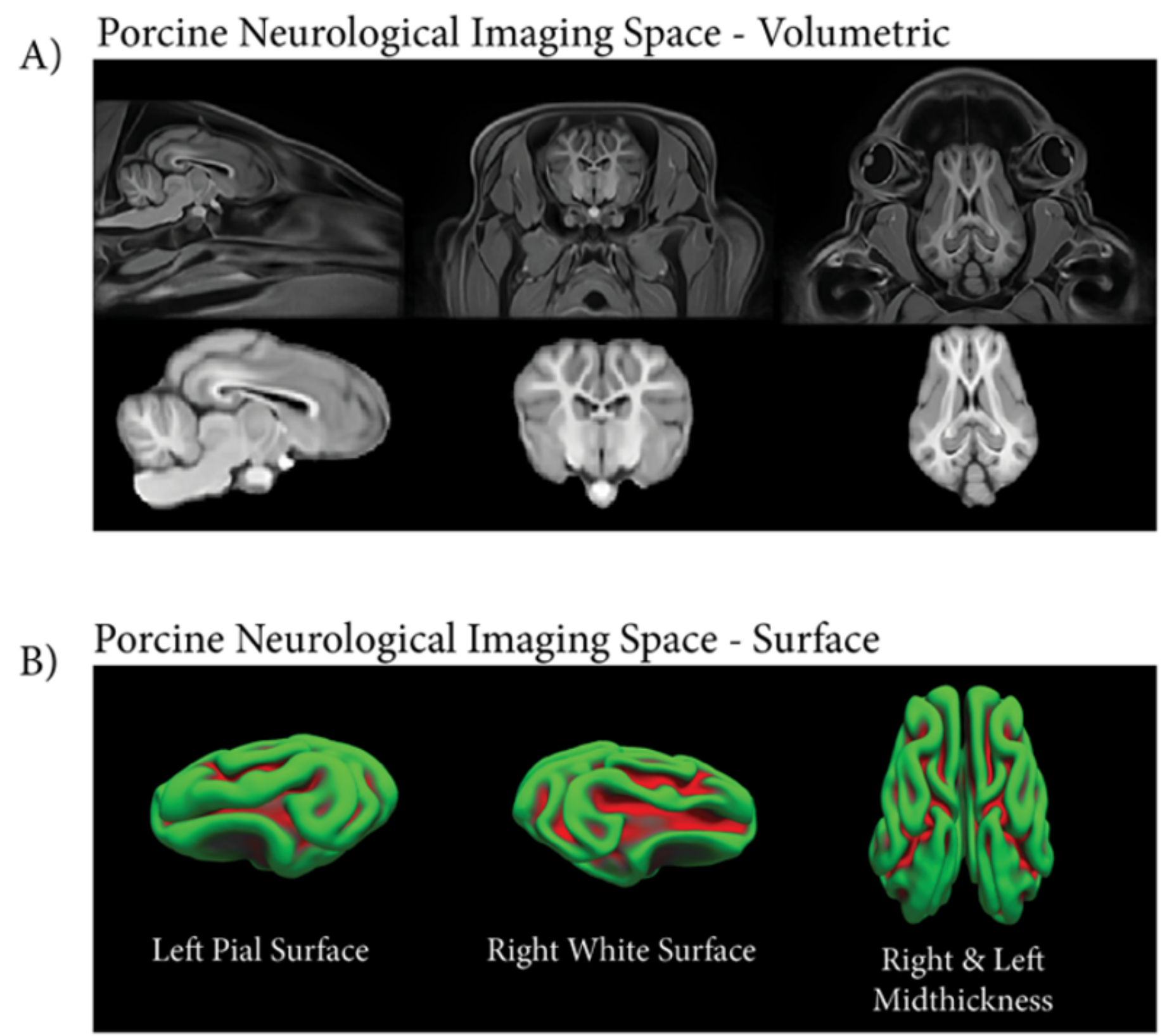

Figure 2

Average Volumetric and Surface Templates of the Porcine Neurological Imaging Space. A. Volumetric full body and brain extracted templates of the Porcine Neurological Imaging Space N=50 (PNI50). Templates were used as a standard space for group-level processing porcine tracts. B. Average surfaces derived from individuals making up the PNI50. The surface used data-driven tractography and blueprints were the 
average mid-thickness surfaces of each hemisphere. Volumetric results in PNI50 space can be projected to the surface and thus enabled for translation using the pig's connectivity blueprint.

\section{The Projection Tracts, Components, and Protocols}
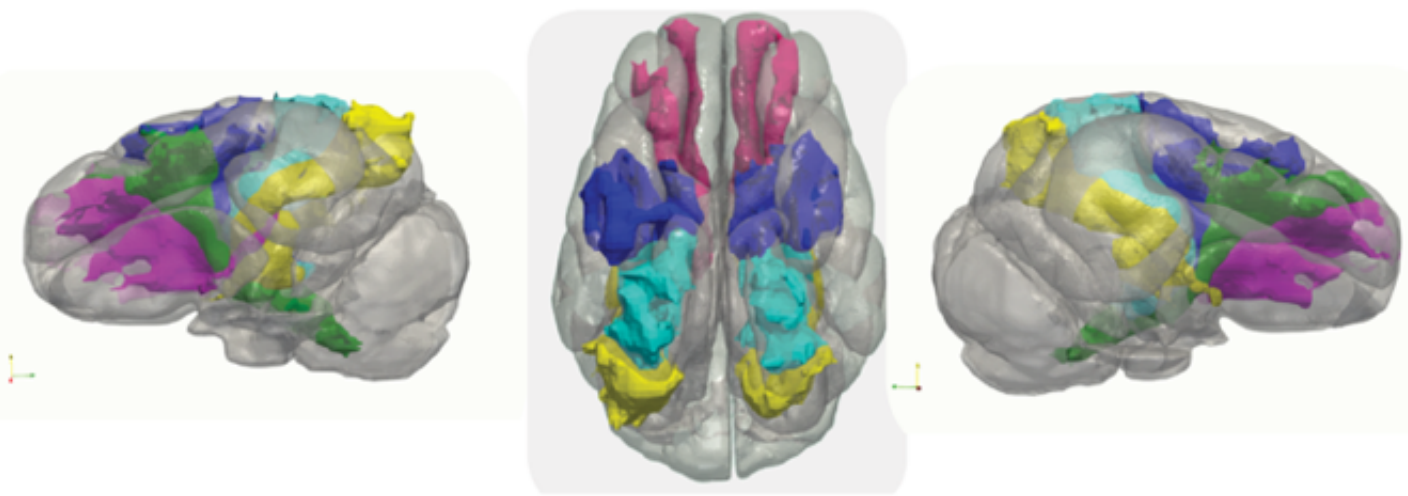

A.

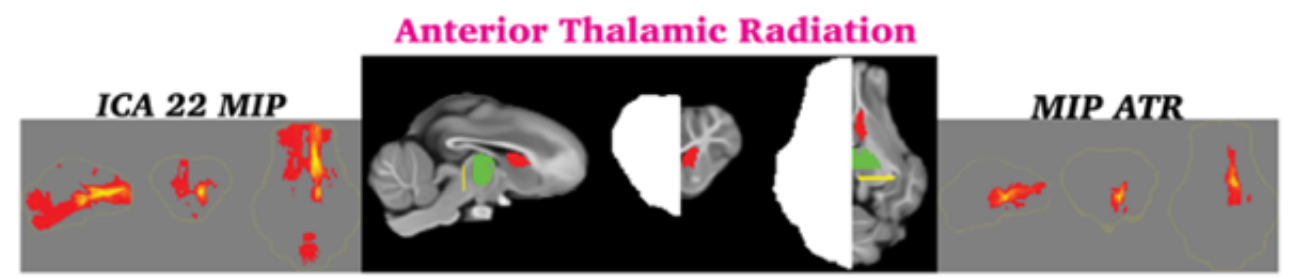

(2) p)te Radiatom

B.

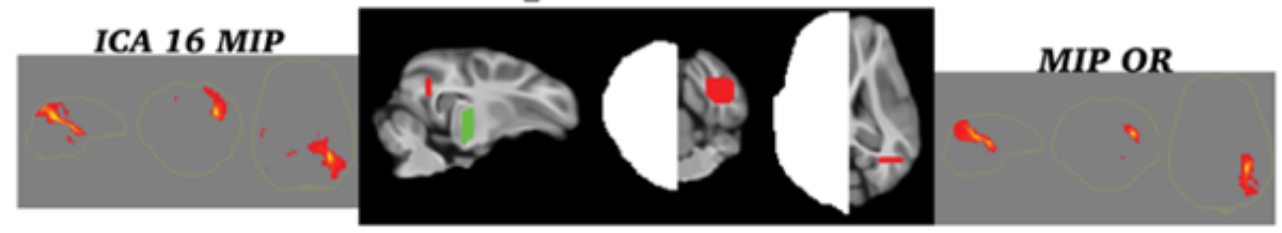

Posterior Thalamic Radiation

C.

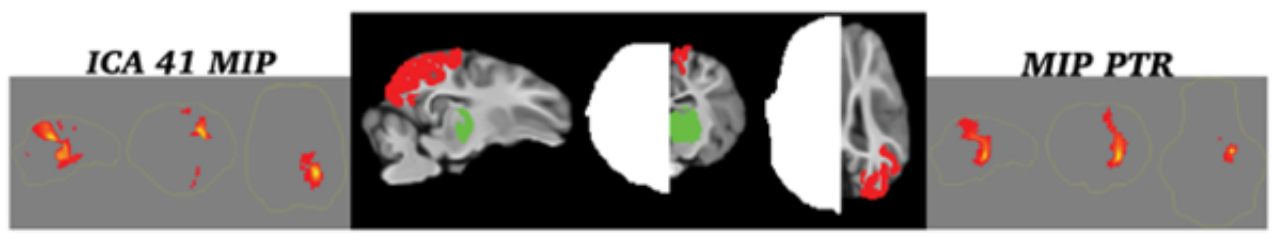

D.

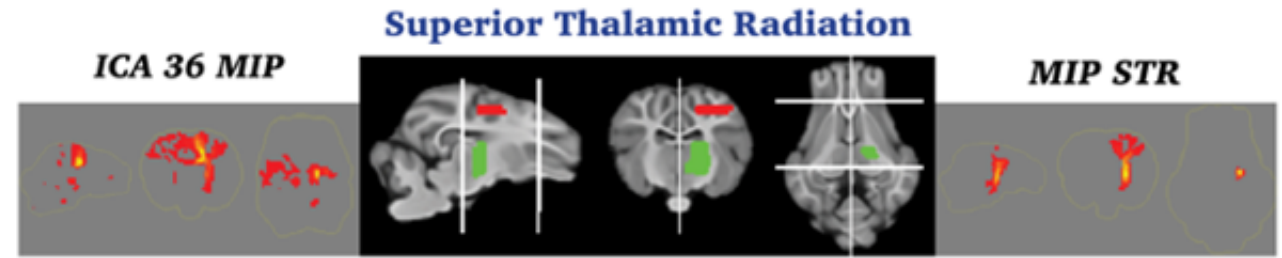

Corticospinal Tract

E.

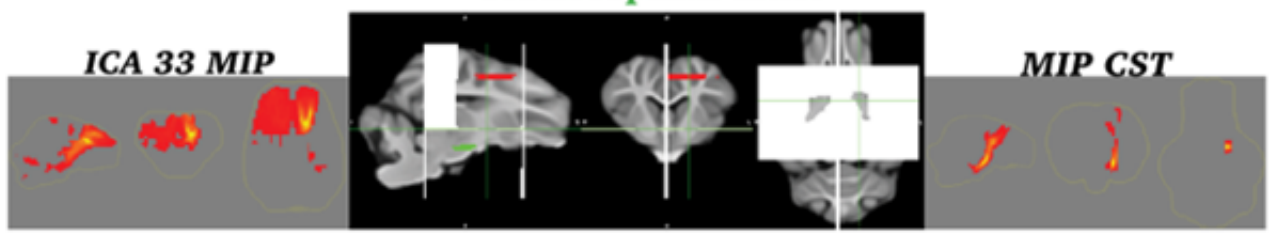

\section{Figure 3}

The Projection Tracts of the pig and human, including the Anterior (ATR), Occipital (OR), Posterior (PTR), and Superior (STR) thalamic radiations and Corticospinal tract (CST) are visualized as 3D reconstructions. A). The maximum intensity projection (MIP) of component $L-22$, the tractography 
protocol used for the final reconstruction, and the MIP of the ATR reconstructed with the mask protocols.

B). The MIP of component L-16, the tractography protocol, and the MIP of the OR. C). The MIP of component L-41, the tractography protocol, and the MIP of the PTR. D). The MIP of component L-36 is associated with the STR, the tractography protocol, and the MIP of the STR. E). The MIP of component L33 , the tractography protocol, and the MIP of the CST.

\section{The Cross-Hemispheric Tracts, Components and Protocols}

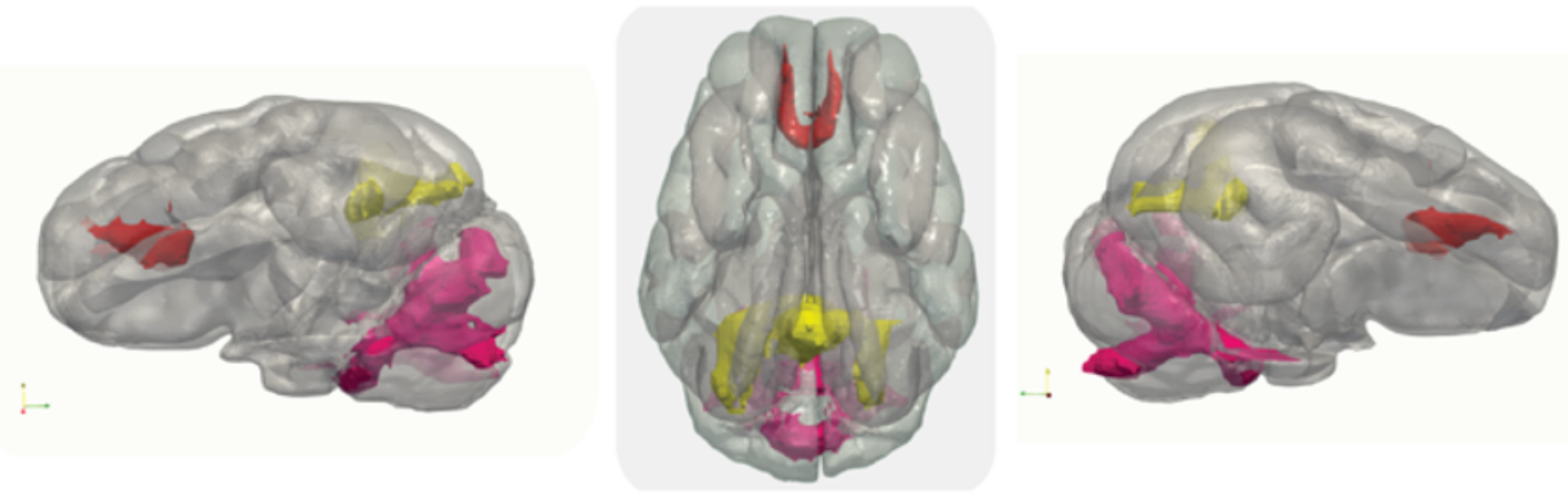

Fore्eps VTajor

A.

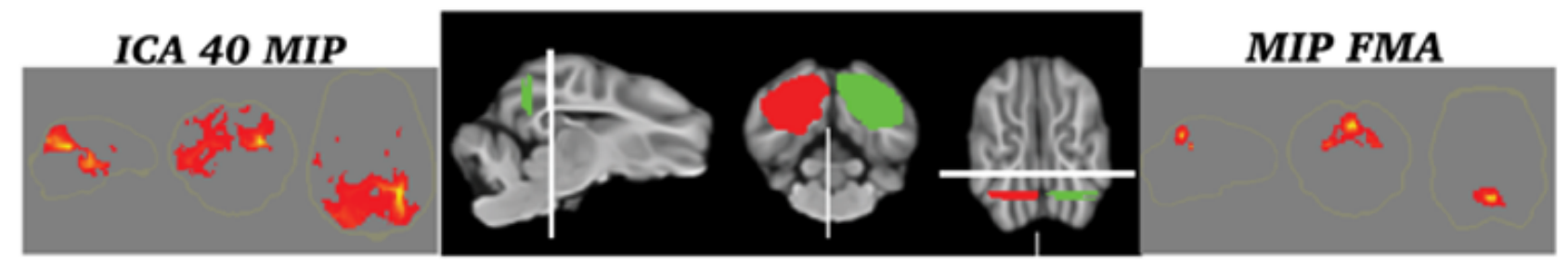

Forceps Minor

B.

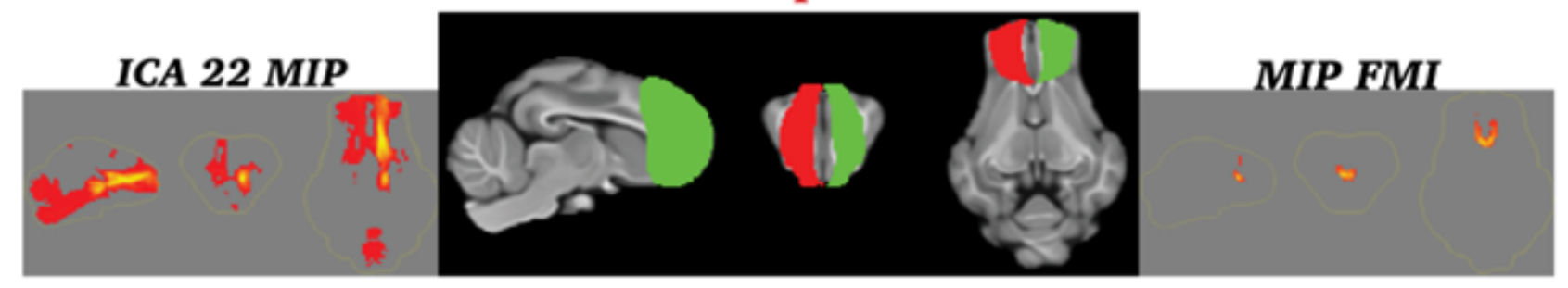

Middle Cerebellar Peduncle

C.

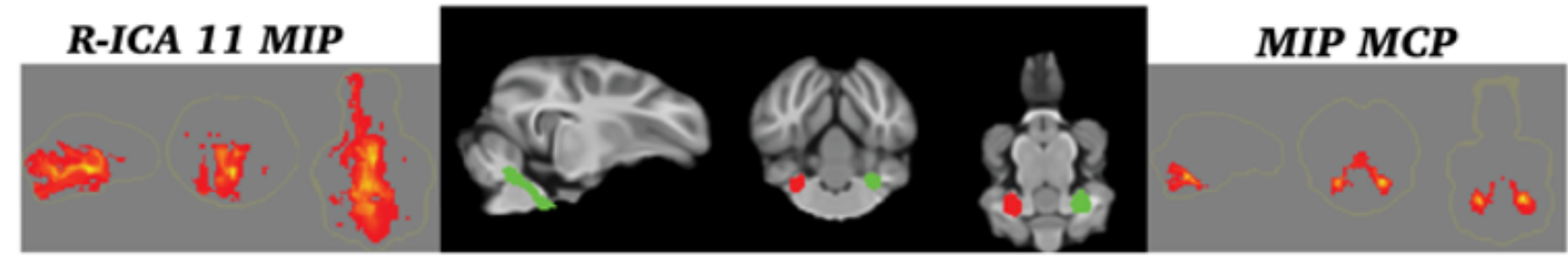

Figure 4 
The Commissural and Cross-Hemispheric Tracts of the pig and human, including the Forceps Major (FMA) and Minor (FMI), and the Middle Cerebellar Peduncle (MCP) reconstructed in 3D. A). The maximum intensity projection (MIP) of component L-40, the tractography protocol used for the final reconstruction, and the MIP of the FMA reconstructed with the mask protocols. B). The MIP of component L-22, the tractography protocol, and the MIP of the FMI. C). The MIP of component R-11, the tractography protocol, and the MIP of the MCP.

\section{The Association Tracts, Components and Protocols}
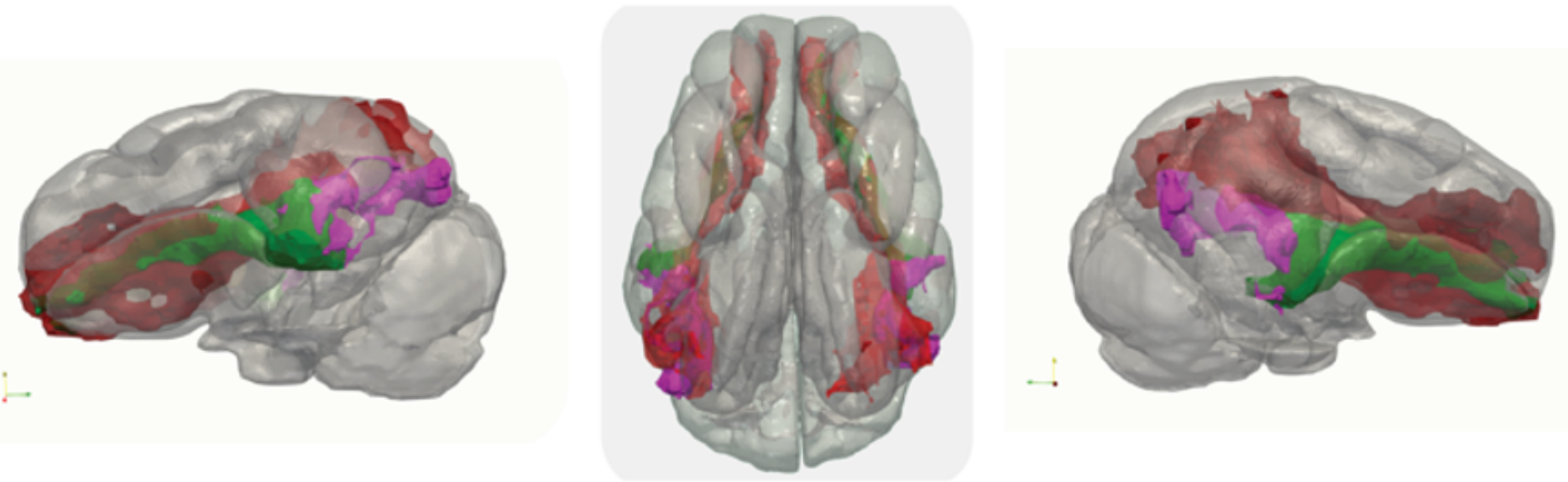

A.

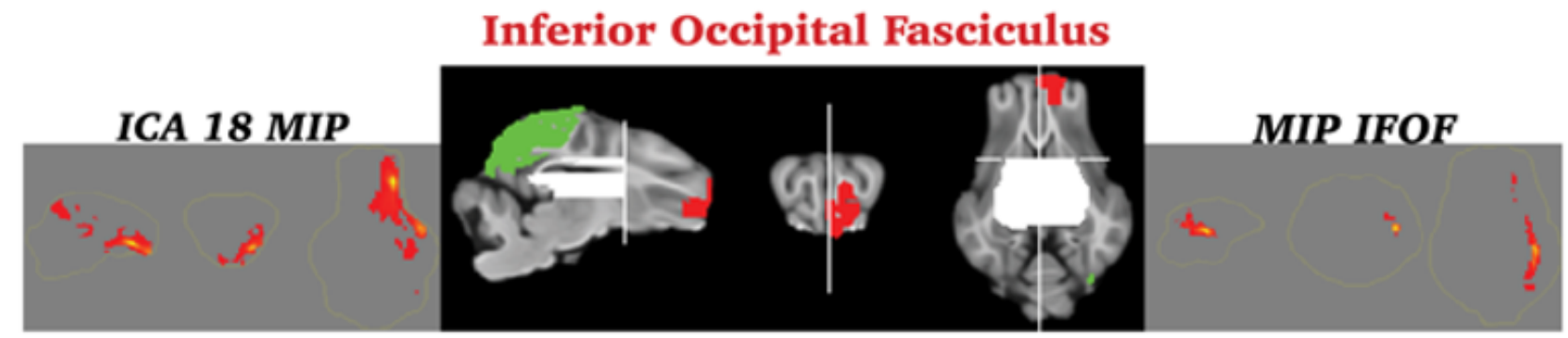

Inferior Longitudinal Fasciculus

B.
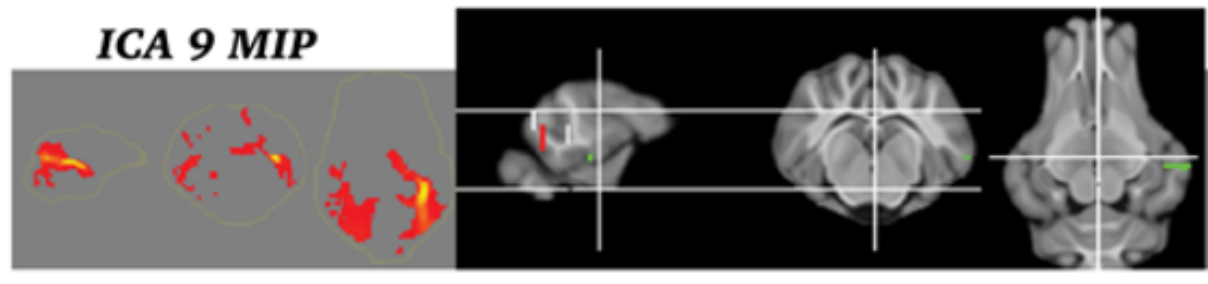

MIP ILF

Uncinate Fasciculus

C.

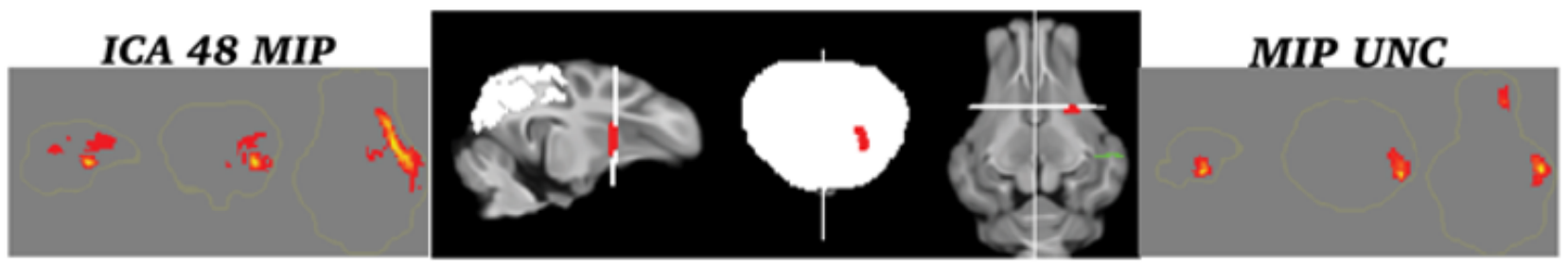

Figure 5 
The Association tracts of the pig and human, including the Inferior-frontal Occipital Fasciculus (IFOF), the Inferior longitudinal Fasciculus (ILF), and the Uncinate Fasciculus (UNC) reconstructed in 3D. A). The maximum intensity projection (MIP) of component $L-18$, the tractography protocol used for reconstruction of the IFOF, and the MIP of the IFOF reconstructed with the mask protocols. B). The MIP of component L-9, the tractography protocol, and the MIP of the ILF. C). The MIP of component L-48, the tractography protocol, and the MIP of the UNC.

\section{The Limbic Tracts, Components and Protocols}
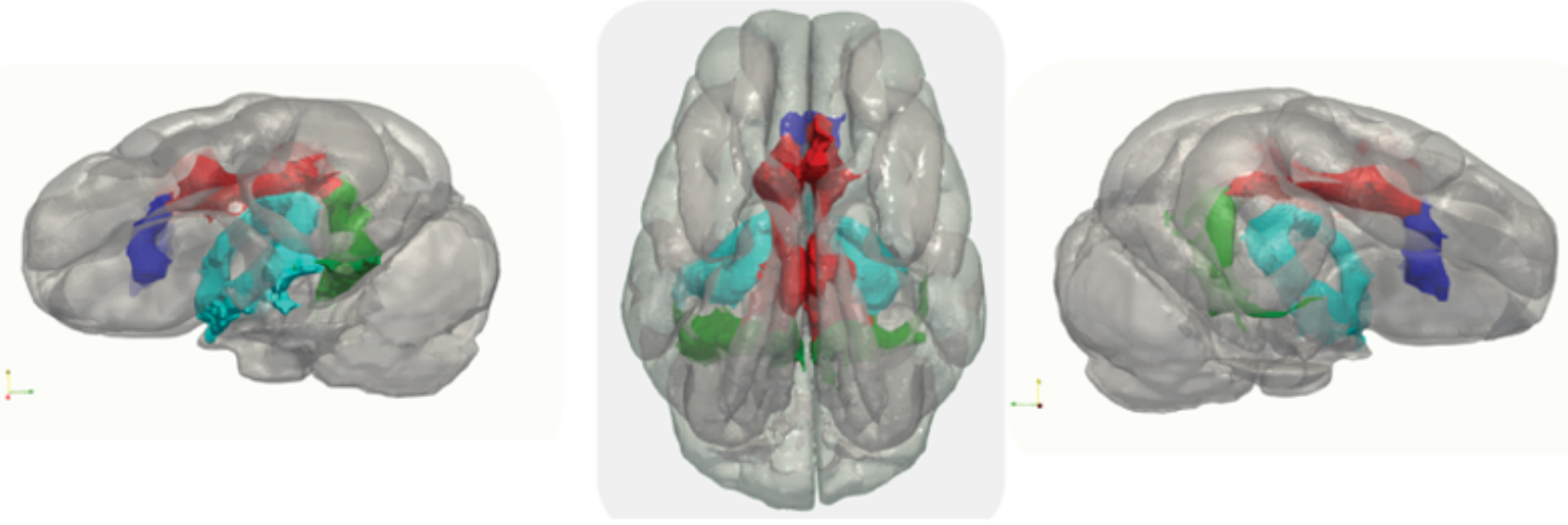

Cingulum Dorsal Bundle

A.

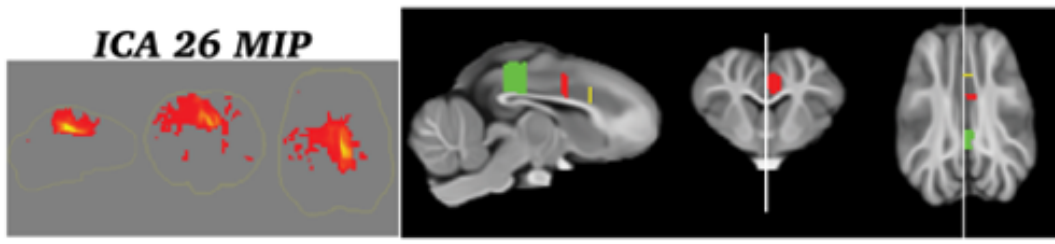

MIP CBD

Pregeneual Cingulum

B.

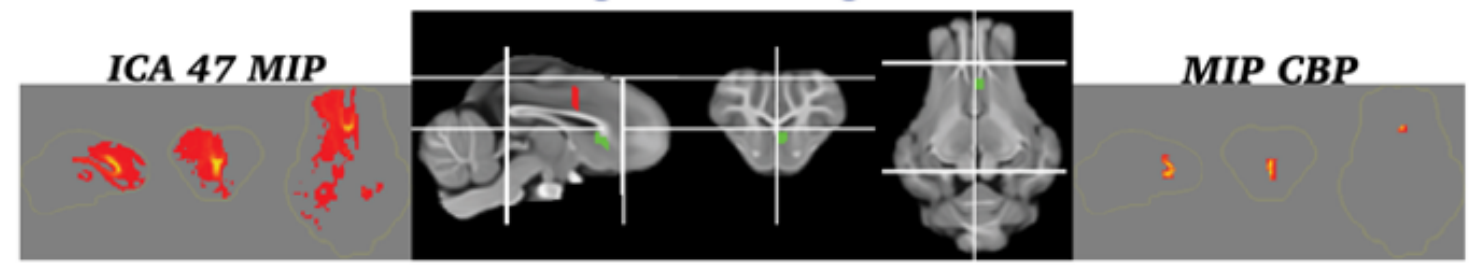

Temporal Cingulum

C.
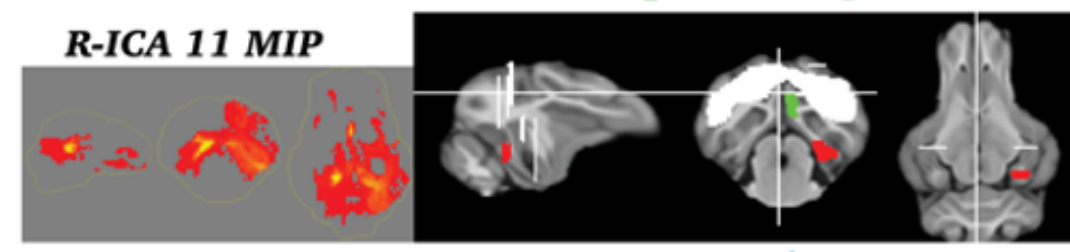

MIP CBT

D.

Fornix

ICA 1 MIP
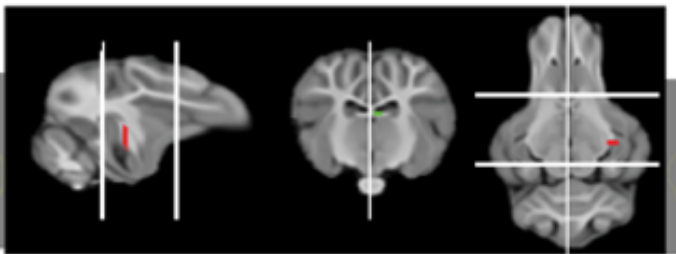

MIP Fx

Figure 6 
The Limbic tracts of the pig and human, including the Cingulum Dorsal Bundle (CBD), The Pregenual Cingulum (CBP), the Temporal Cingulum (CBT), and the Fornix (Fx) reconstructed in 3D. A). The maximum intensity projection (MIP) of component L-26, the tractography protocol used for reconstruction of the CBD, and the MIP of the CBD reconstructed with the mask protocols. B). The MIP of component L-47, the tractography protocol, and the MIP of the CBP. C). The MIP the component R-11, the tractography protocol, and the MIP of the CBT. D). The MIP of component L-1, the tractography protocol, and the MIP of the Fx.

A. Symmetry of Porcine White Matter Cortical Projections

B. Cross Species Whole Cortex Connectivity Fingerprints
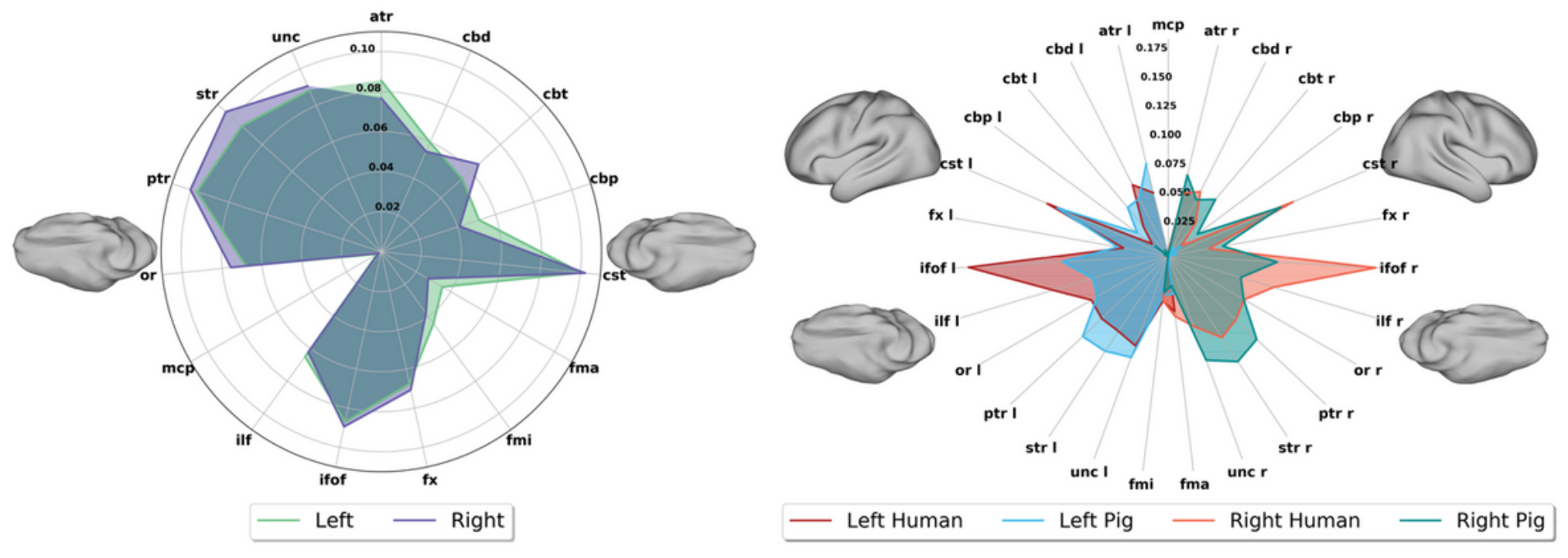

\section{Figure 7}

A). Symmetry shown via the whole cortex fingerprint of the left and right hemispheres shows the pig to have similar connectivity within each hemisphere. Of note, the prefrontal Forceps Minor (FMI) and Cingulum Pregenual Bundle(CBP) show a bias to the left hemisphere, while the superior and posterior thalamic radiations (STR/PTR) have more robust connectivity in the right hemisphere. B). Connectivity fingerprints of the left and right cortex in both species. Connectivity across hemispheres is overall symmetric in both species. The connectivity fingerprint shows which tracts are vital in driving the $\mathrm{KL}$ divergence across the pig and human cortex. Of particular note are the increased human connections to the Inferior Fronto-Occipital Fasciculus (IFOF), Inferior Longitudinal Fasciculus (ILF), and Cingulum Dorsal Bundle (CBD). The pig cortex had increased connectivity with the Cingulum Pregenual Bundle (CBP), Uncinate Fasciculus (UNC), and the STR and PTR. 


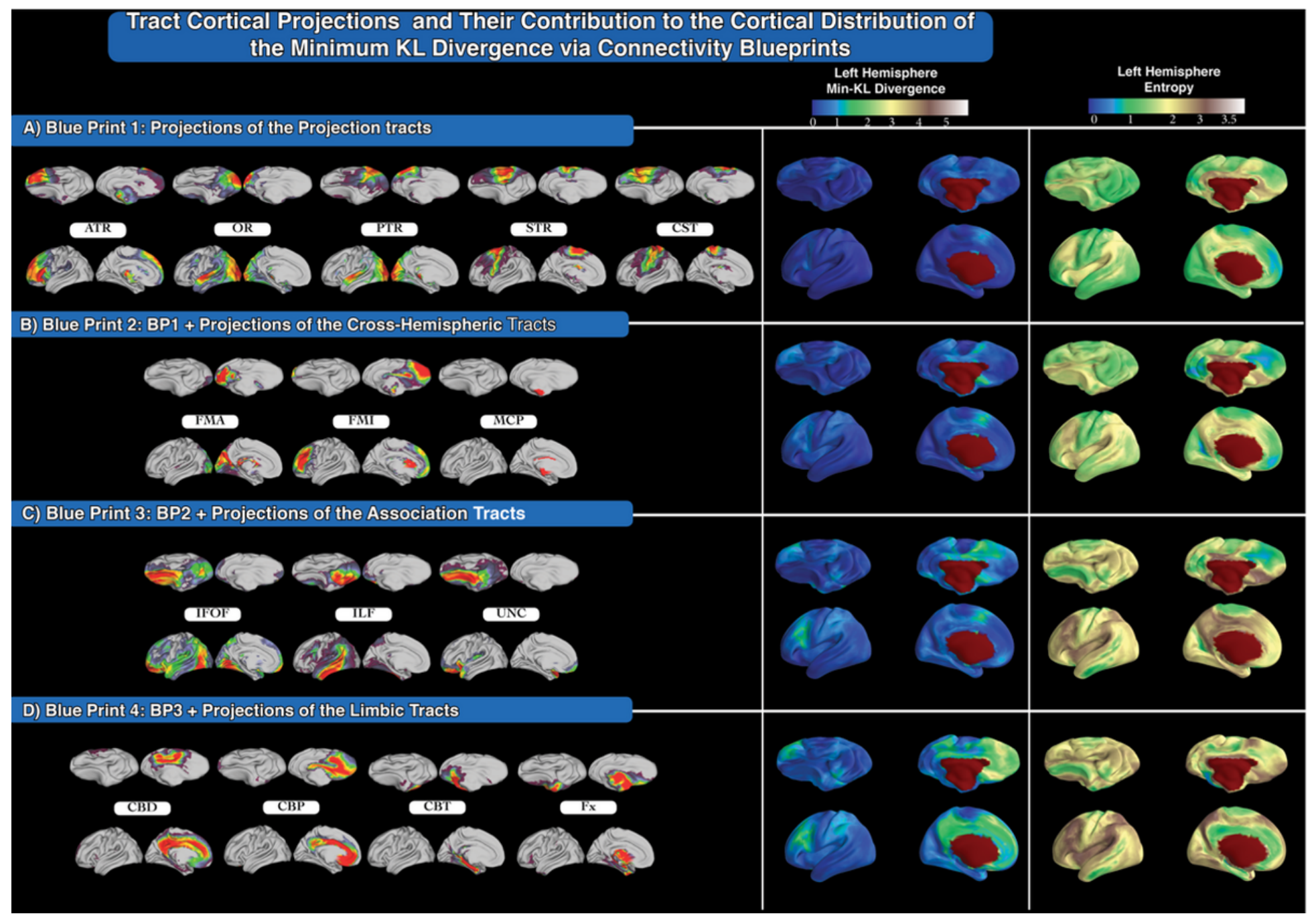

Figure 8

A). Blueprint 1 contains the cortical tract projections of the Anterior (ATR), Occipital (OR), Posterior (PTR), Superior (STR), and Corticospinal tract (CST). The minimum KL divergence is plotted to the left showing slight peaks in regions of the ATR and CST in both species. The distribution of entropy or the diversity of tracts each vertex connects to displays a distribution independent of the $\mathrm{KL}$ divergence B). Blueprint 2 contains the cortical tract projections of blueprint 1 and adds the Forceps Major (FMA), Forceps Minor (FMI), and Middle Cerebellar Peduncle (MCP). C). Blueprint 3 adds the Inferior Fronto-occipital fasciculus (IFOF), Inferior Longitudinal Fasciculus, and Uncinate Fasciculus to blueprint 2. Doing so shifts the maximum of the min-KL divergence into the parietal and frontal lobes of both species. D). Blueprint 4 adds the Cingulum Dorsal Bundle, the Pregenual Cingulum, Temporal Cingulum, and Fornix to blueprint 3. The cingulum fibers in the human form a continuum not found in the pig, and as a result, the min-KL divergence shifts along the cingulum body and medial frontal lobe in both species. This is evidenced by a drop in entropy along the limbic fibers in the human brain, not present in the pig. This suggests the continuum of cingulum tract projections present in the human, but not the pig strongly increases the $\mathrm{KL}$ divergence across species. 


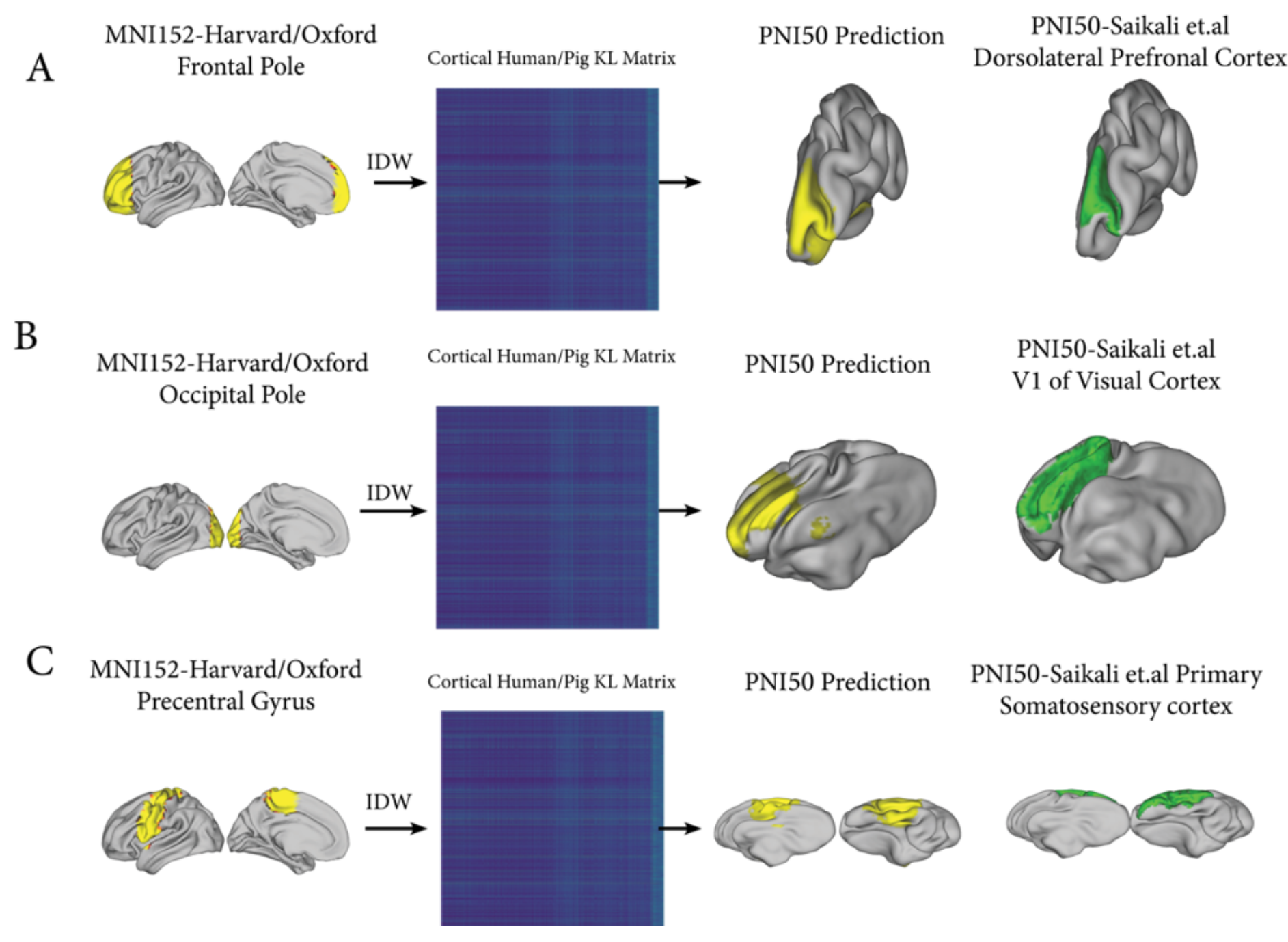

\section{Figure 9}

Spatial predictions of human regions of interest onto the porcine cortex using the $\mathrm{KL}$ similarity matrix calculated between the pig and human blueprint 4. A). The frontal pole, as defined in the Harvard/Oxford atlas, interpolated and predicted onto the pig (yellow) and the Saikali et al. DLPFC (green) in PNI50 space. B). The occipital pole in the Harvard/Oxford atlas interpolated and predicted onto the pig (yellow) and the ground truth V1 mask of Saikali et al. atlas in PNI50 space. C). The precentral gyrus, as defined in the Harvard/Oxford atlas, interpolated and predicted onto the pig (yellow) and the ground truth primary somatosensory cortex of the Saikali et al. atlas in PNI50 space. 
PNI50-Saikali et.al

A

Dorsolateral Prefronal Cortex

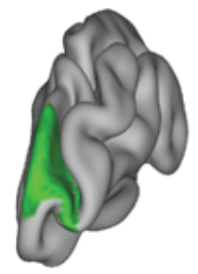

B

\begin{abstract}
PNI50-Saikali et.al V1 of Visual Cortex
\end{abstract}

\section{$\stackrel{\text { IDW }}{\longrightarrow}$}
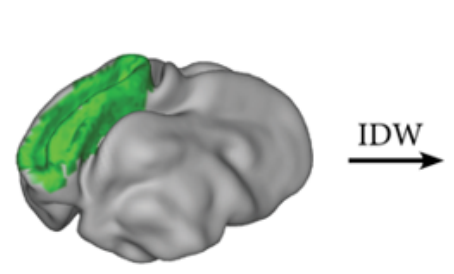

Cortical Human/Pig KL Matrix

C PNI50-Saikali et.al Primary Somatosensory cortex

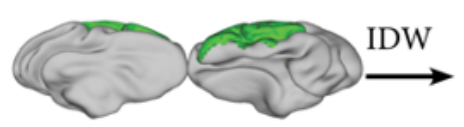

Cortical Human/Pig KL Matrix

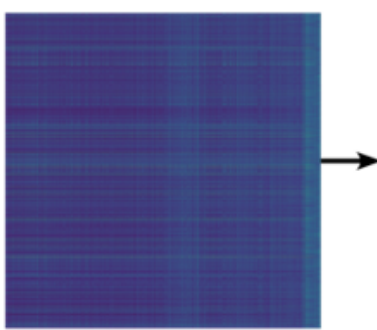

MNI152 Prediction

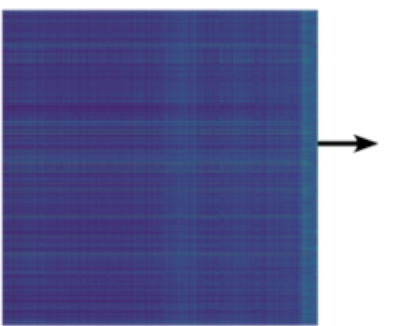

Cortical Human/Pig KL Matrix
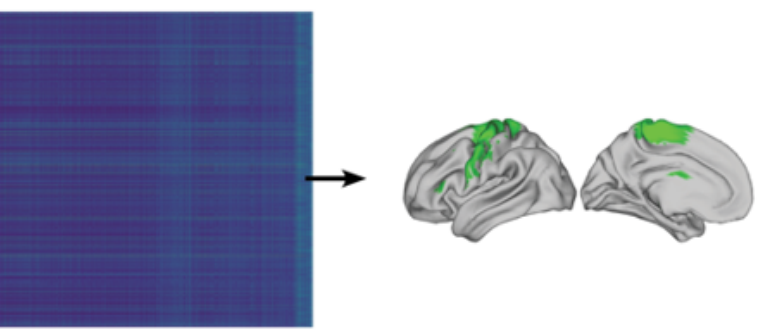

MNI152 Prediction

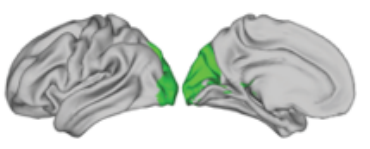

MNI152 Prediction
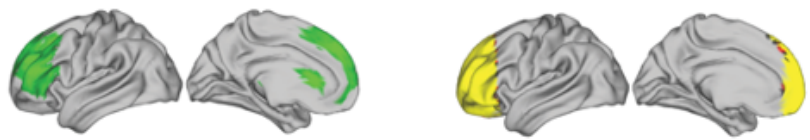

MNI152-Harvard/Oxford Occipital Pole

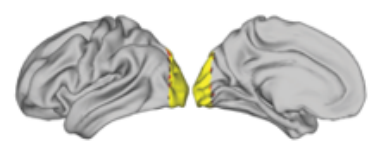

MNI152-Harvard/Oxford Precentral Gyrus

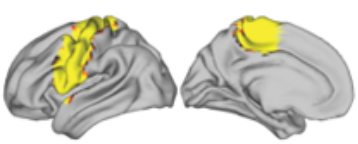

\section{Figure 10}

The inverse of figure 9, predictions of pig regions of interest onto the human cortex using the $\mathrm{KL}$ similarity matrix calculated between the pig and human blueprint 4. A). The Saikali et al. DLPFC (green) in PNI50 space interpolated and predicted onto the human surface (green) and the frontal pole as defined in the Harvard/Oxford atlas (yellow). B). The Saikali et al. V1 of the visual cortex (green) in PNI50 space interpolated and predicted onto the human surface (green) and the occipital pole as defined in the Harvard/Oxford atlas (yellow). C). The Saikali et al. Primary Somatosensory Cortex (green) in PNI50 space interpolated and predicted onto the human surface (green) and the precentral gyrus as defined in the Harvard/Oxford atlas (yellow).

\section{Supplementary Files}

This is a list of supplementary files associated with this preprint. Click to download.

- supplementarymaterial.docx 